\title{
Comparison of brain connectomes by MRI and genomics and its implication in Alzheimer's disease
}

\author{
Young Jae Woo ${ }^{1}$, Panos Roussos ${ }^{1,2}$, Vahram Haroutunian ${ }^{2,3}$, Pavel Katsel2 ${ }^{2}$, Samuel Gandy ${ }^{2,4}$, Eric E. Schadt ${ }^{1,5}$,
} Jun Zhu ${ }^{1,5^{*}}$ (D) and Alzheimer Disease Neuroimaging Initiative (ADNI)

\begin{abstract}
Background: The human brain is complex and interconnected structurally. Brain connectome change is associated with Alzheimer's disease (AD) and other neurodegenerative diseases. Genetics and genomics studies have identified molecular changes in AD; however, the results are often limited to isolated brain regions and are difficult to interpret its findings in respect to brain connectome. The mechanisms of how one brain region impacts the molecular pathways in other regions have not been systematically studied. And how the brain regions susceptible to AD pathology interact with each other at the transcriptome level and how these interactions relate to brain connectome change are unclear.

Methods: Here, we compared structural brain connectomes defined by probabilistic tracts using diffusion magnetic resonance imaging data in Alzheimer's Disease Neuroimaging Initiative database and a brain transcriptome dataset covering 17 brain regions.

Results: We observed that the changes in diffusion measures associated with AD diagnosis status and the associations were replicated in an independent cohort. The result suggests that disease associated white matter changes are focal. Analysis of the brain connectome by genomic data, tissue-tissue transcriptional synchronization between 17 brain regions, indicates that the regions connected by $\mathrm{AD}$-associated tracts were likely connected at the transcriptome level with high number of tissue-to-tissue correlated (TTC) gene pairs $(P=0.03)$. And genes involved in $T$ C gene pairs between white matter tract connected brain regions were enriched in signaling pathways $\left(P=6.08 \times 10^{-9}\right)$. Further pathway interaction analysis identified ionotropic glutamate receptor pathway and Toll receptor signaling pathways to be important for tissue-tissue synchronization at the transcriptome level. Transcript profile entailing Toll receptor signaling in the blood was significantly associated with diffusion properties of white matter tracts, notable association between fractional anisotropy and bilateral cingulum angular bundles $\left(P_{\text {permutation }}=1.0 \times 10^{-2}\right.$ and $4.9 \times 10^{-4}$ for left and right respectively).

(Continued on next page)
\end{abstract}

\footnotetext{
* Correspondence: jun.zhu@mssm.edu

Data used in preparation of this article were obtained from the Alzheimer's Disease Neuroimaging Initiative (ADNI) database (adni.loni.usc.edu). As such the investigators within the ADNI contributed to the design and implementation of ADNI and/or provided data but did not participate in the analysis or writing of this report. A complete listing of ADNI investigators can be found at http://adni.loni.usc.edu/wp-content/uploads/how_to_apply/

ADNI_Acknowledgement_List.pdf.

'Department of Genetics and Genomic Sciences, Icahn School of Medicine at Mount Sinai, New York, NY 10029, USA

${ }^{5}$ Sema4, Stamford, CT 06902, USA

Full list of author information is available at the end of the article
}

(c) The Author(s). 2020 Open Access This article is distributed under the terms of the Creative Commons Attribution 4.0 International License (http://creativecommons.org/licenses/by/4.0/) which permits unrestricted use, distribution, and reproduction in any medium, provided you give appropriate credit to the original author(s) and the source, provide a link to the Creative Commons license, and indicate if changes were made. The Creative Commons Public Domain Dedication waiver (http://creativecommons.org/publicdomain/zero/1.0/) applies to the data made available in this article, unless otherwise stated. 
(Continued from previous page)

Conclusions: In summary, our study suggests that brain connectomes defined by MRI and transcriptome data overlap with each other.

Keywords: Alzheimer's disease, Diffusion tensor imaging, Transcriptome, Post-mortem brain, Imaging transcriptomics, Toll-like receptor signaling

\section{Background}

Neural connections comprising a nervous system are often described in complicated anatomical networks. Much of the human brain connectome has been assessed using magnetic resonance imaging (MRI) where functional MRI and diffusion MRI (dMRI) can measure correlated neural activity and structural connectivity of the brain in vivo, respectively [1, 2]. Various neurological diseases such as Alzheimer's disease (AD) are associated with disruption of the brain connectome and studies show that the course of $\mathrm{AD}$ continuum is associated with the changes in brain network architecture [3-5]. Although our knowledge regarding the connectome changes in AD is abundant, understanding the molecular consequences or causes of brain connectome changes is lacking.

Gene expression signatures carry important information for understanding structural and functional brain connectivity. It has been shown that the connectivity in rodent brains can be predicted from mouse brain expression data $[6,7]$. Brain connectivity based on blood-oxygen-leveldependent signals at a resting state is significantly associated with correlations between gene expression of human brain segments [8]. However, the transcriptomic studies of $\mathrm{AD}$ are often limited to isolated brain regions such as the hippocampus or dorsolateral prefrontal cortex alone and are difficult to interpret its findings in respect to the brain connectome when its relation is not examined together $[9,10]$. The mechanisms of how one brain region impacts molecular pathways in other regions, especially how the brain regions susceptible to AD pathology interact with each other at the transcriptome level, have not been systematically studied.

Here, we performed imaging-transcriptomic study analyses of brain connectomes based on dMRI imaging data from Alzheimer's Disease Neuroimaging Initiative (ADNI) and a brain transcriptome dataset covering 17 brain regions [11-13]. Unlike traditional imaging genetic association analyses, where the goal is to identify the relationship between genetic variation and the changes in neurological traits $[14,15]$, the analyses here focused in spatial correlations between gene expression and structural brain connectivity. We hypothesize that different brain regions are synchronized at the molecular level (genomic connectome), partially facilitated by white matter tracts (structural connectome). Dysfunction of genomic connectome may associate with neurological diseases and reflect genetic propensity underlying AD etiology. To test our hypothesis, we (1) identified white matter tracts associated with AD based on dMRI and replicated them in an independent cohort [16], (2) identified brain regions connected by white matter tracts, (3) compared structural brain connections and genomic brain connections defined as tissue-to-tissue correlations (TTCs) at the transcription level, and (4) identified biological pathways involved in TTCs in structurally connected brain regions $[17,18]$.

\section{Methods \\ Neuroimaging analysis}

Data used in the preparation of this study were obtained from the ADNI database (http://adni.loni.usc.edu). The ADNI was launched in 2003 as a public-private partnership, led by Principal Investigator Michael W. Weiner, MD. The primary goal of ADNI has been to test whether serial magnetic resonance imaging (MRI), positron emission tomography (PET), other biological markers, and clinical and neuropsychological assessment can be combined to measure the progression of mild cognitive impairment (MCI) and early Alzheimer's disease (AD). There were 232 ADNI2 subjects and 621 ADNI3 subjects with both $\mathrm{T} 1$ and diffusion-weighted MRI images at baseline when we downloaded the data on October 2018 from the Laboratory of Neuro-Imaging (http:// adni.loni.usc.edu) [11]. All images were converted from DICOM to NIFTI using DCM2NII software (University of South Carolina, SC, USA) [19]. All anatomical regions of interests (ROIs) were segmented using FreeSurfer 6.0, 64-bit version (Massachusetts General Hospital, MA, USA) [20]. The FreeSurfer pipeline included motion correction of volumetric T1-weighted imaged, stereotaxic space transformation, intensity non-uniformity correction, removal of non-brain tissue, tessellation of gray/ white matter boundaries via surface modeling, automatic topology correction, and surface deformation followed by intensity gradient that optimally defined tissue borders where the greatest shift in intensity defined the transition into the other tissue. Image outputs were visually checked for each subject. Segmentation of ROIs was conducted based on "Desikan-Killiany" cortical atlas [21]. Diffusion-weighted images were preprocessed using FSL 5.0.10 (Wellcome Center, Oxford, UK) [22]. Diffusion imaging pipeline included brain extraction, susceptibility-induced distortion correction, eddy current 
and motion correction, individuals' axial diffusivity $(A x D)$, radial diffusivity (RD), mean diffusivity (MD), and fractional anisotropy (FA) estimation, and diffusion uncertainty map calculation using BEDPOSTX [23-25]. Probabilistic tractography was performed using TRActs Constrained by UnderLying Anatomy (TRACULA), and 18 tracts were derived (Massachusetts General Hospital, MA, USA) [26]. The 18 tracts are forceps major, forceps minor, left anterior thalamic radiations (L-ATR), left cingulum-angular bundle (L-CAB), left cingulum-cingulate gyrus (L-CCG), left corticospinal tract (L-CST), left inferior longitudinal fasciculus (L-ILF), left superior longitudinal fasciculus parietal (L-SLFP), left superior longitudinal fasciculus temporal (L-SLFT), left uncinate fasciculus (L-UNC), right anterior thalamic radiations (R-ATR), right cingulum-angular bundle (R-CAB), right cingulum-cingulate gyrus (R-CCG), right corticospinal tract (R-CST), right inferior longitudinal fasciculus (RILF), right superior longitudinal fasciculus parietal (RSLFP), right superior longitudinal fasciculus temporal ( $R$ SLFT), and right uncinate fasciculus (R-UNC). For each tract, volume, average length, mean $\mathrm{AxD}$, mean $\mathrm{RD}$, mean $\mathrm{MD}$, and mean FA were calculated.

Imaging data for ADNI2 and ADNI3 cohorts were processed under identical procedure. After all quality checks which include both systematic error checks and visual inspections, 593 out of 621 ADNI3 subjects and 220 out of 232 ADNI2 subjects successfully finished all imaging processes. Among 593 ADNI3 subjects, 550 subjects had qualifying clinical measures where age and gender were available and diagnosis record was within 60 days of scan date. Among 220 imaging processed subjects in ADNI2, 210 subjects had matching relevant phenotypes also within 60 days of scan date. Fifty-one subjects overlapped between ADNI3 and ADNI2 cohorts that had finished all the image processing and phenotype matched with scan date. Therefore, we omitted overlapping subjects from ADNI3 and analyzed 499 subjects for ADNI3 as discovery cohort and 210 ADNI2 subjects as the replication cohort. Association between AD diagnosis and diffusion measures of each tract (volume, average length, AxD, RD, MD, FA) was examined via linear model adjusted for age, gender, and total brain volume. Outliers that were 3.5 SD away from the mean were removed from the model, and all statistics were false discovery rate (FDR) adjusted for multiple comparisons. Effect sizes $(\beta)$ of associations with $A D$ (diffusion measure $y=\beta^{*} \mathrm{AD}$ after adjusting covariates) was also calculated from the models.

\section{Reach probability calculation}

The probability of tract reaching a Desikan-Killiany atlas defined grey matter ROIs at its white matter border was estimated. One voxel deep grey matter mask that is neighboring white matter was derived for all 82 ROIs and defined as ROI target masks. Path distribution for each tract was trimmed to include white matter and ROI target masks only. The probability of tract reaching ROI at its white matter boundary was calculated by dividing the number of paths passing through each voxel by the total path number in trimmed tract. Finally, the normalized probabilities within ROI target masks were averaged for all ROIs and this was repeated for each tract (Additional file 1: Figure S1A). These were defined as "reach probability" of tract connecting to grey matter ROIs (18 tracts towards 82 ROIs). The non-zero reach probability followed an extreme value distribution (Additional file 1: Figure S2A). We defined connections based on the empirical cumulative distribution function reflection point (reach probability $=0.002$ ). At the cutoff, 203 of ROI-tract pairs were connected by a white matter tract. This effectively isolated some ROIs to specific tracts such as L-hippocampus was connected to L-CAB but not with L-SLFT or L-SLFP (Additional file 2: Table S1).

\section{Tissue-to-tissue correlated gene identification}

Post-mortem brain tissues curated by Mount Sinai Hospital were analyzed where gene expression for 17 brain regions limited to the left hemisphere were available $[12,13]$. The transcriptome data was made up of maximum 63 subjects, and any two brain regions were shared by $30-51$ subjects (Additional file 2: Table S2). The methods and cohort characteristics for this dataset have been described in detail $[12,13]$. The 17 brain regions were frontal pole (FP), occipital visual cortex (OVC), inferior temporal gyrus (ITG), middle temporal gyrus (MTG), superior temporal gyrus (STG), posterior cingulate cortex (PCC), anterior cingulate cortex (ACC), parahippocampal gyrus (PHG), temporal pole (TP), precentral gyrus (PCG), inferior frontal cortex (IFC), dorsolateral prefrontal cortex (DLPFC), superior parietal lobule (SPL), prefrontal cortex (PFC), caudate nucleus $(\mathrm{CN})$, hippocampus (HIP), and putamen (PUT) (Additional file 2: Table S2). The gene expression values were adjusted for age, sex, post-mortem interval, $\mathrm{pH}$, ethnicity, and Braak staging scores. The adjustment removed potential batchdriven gene-gene correlations, such as both genes were up in an ethnic group or disease diagnosis group, but enhanced gene-gene correlations that were consistent among ethnic groups or disease diagnosis groups, and etc. Spearman correlation was used in identifying TTC gene pairs between 136 pairs of brain regions $(17 \times 16 / 2)$. Significant TTC gene pairs were identified at genome-wide threshold $P$-value $<1 \times 10^{-8}$ as defined in previous TTC study [17]. Significant TTC gene pairs were counted for 136 brain region pairs (Additional file 2: Table S3).

\section{Bipartite clustering}

Significant TTC signals were discretized as binary values, and Barber's modularity was maximized which identifies 
two-mode networks of disjoint gene sets such that interaction only occurs with genes of another brain region [27]. LPAb+ algorithm outperforms other methods for bipartite networks $[28,29]$, and we utilized its two-stage procedure where first "bottom-up" step propagates labels iteratively to maximize node-by-node modularity and second "top-down" step joins modules together to increase network modularity [30]. Different random initialization of node selection was performed five times for all 136 ROI pairs and confirmed that the maximized modularity converged to same optimal solution. For each ROI pair, bipartite modules with more than 1000 interactions (TTC gene pairs) were selected and genes within modules were pooled for each tissue before conducting pathway enrichment analysis.

\section{Pathway enrichment analysis}

Curated pathways from Protein Analysis Through Evolutionary Relationships (PANTHER) database v.14.1 were analyzed [31]. Among 177 curated pathways available, eight pathways made up of drosophila-specific pathways were omitted (P06209, P06211, P06212, P06213, P06214, P06215, P06216, P06217). The Fisher exact test was performed to assess overrepresentation of our gene lists in each pathway, and all human genes $(n=20,996)$ were used as background. All pathway enrichment was corrected for FDR.

In order to infer broad biological insight from pathways overrepresented in the genes involved in TTC gene pairs, we created eight pathway categories that are biosynthesis, signaling, disease, physiology, development, gene regulation, metabolism, and catabolism for which detailed group identity for each pathway is listed in Additional file 2: Table S4. As post hoc analysis, after observing that a large number of associations were part of signaling pathway subgroup, we further divided signaling pathways into synaptic signaling, immune signaling, synaptic immune signaling, endocrine signaling, and unclassified (Additional file 2: Table S4). If the synthesized end product or degraded starting material served as a ligand in any synaptic, immune, or endocrine signaling, they were assigned as such (adrenaline and noradrenaline biosynthesis, aminobutyrate degradation, androgen/estrogen/progesterone biosynthesis, cobalamin biosynthesis, phenylethylamine degradation, vitamin $\mathrm{B}_{6}$ metabolism, gamma-aminobutyric acid synthesis, histamine synthesis, vasopressin synthesis, vitamin D metabolism and pathway, bupropion degradation, nicotine degradation). Oxidative stress response (P00046) was categorized as immune signaling.

\section{Pathway interaction analysis}

For a ROI pair, molecular pathways significantly enriched in genes involved in TTC gene pairs were binarized for each ROIs (FDR < 0.05) (Fig. 4). Binarized pathway associations were matched between ROI1 and ROI2 that are paired in bipartite clustering step and were transformed into adjacency matrix (Additional file 1: Figure S3A). This defines pathway interaction between ROI pairs. There were three types of ROI pairs: (1) ROI pairs not connected by tracts (not-bound), (2) ROI pairs bound by tracts (tract-bound), and (3) ROI pairs bound by AD-associated tracts (AD-tract-bound). Among 136 ROI pairs, there were 72 not-bound, 64 tract-bound, and $43 \mathrm{AD}$-tractbound. Proportion of pathway interactions in each group was calculated by normalizing the summed adjacency matrices by the number of ROI pairs (Additional file 1: Figure S3B). The chi-square test was performed comparing the proportion of pathway interactions for the tract-bound and AD-tract-bound groups. Both comparisons were compared against the not-bound group (Additional file 1: Table S5-6). The $P$-values were $-\log _{10}$ transformed and were hierarchically clustered using Ward's method for further analysis.

\section{Blood expression analysis}

The ADNI study collected whole blood samples for 811 subjects at baseline, which were processed using Qiagen PAXgene Blood RNA Kit (Germantown, MD, USA) [32]. Gene expression was profiled using Affymetrix Human Genome U219 Array (Affymetrix, Santa Clara, CA, USA) and was preprocessed using the Robust Multi-chip Average normalization method [33]. All quality check (QC) procedures were performed by ADNI Genetics Core which include RNA QC using Nanodrop and Agilent Bioanalyzer, overall array assay QC using Affymetrix Expression Console software and Partek Genomic Suite 6.6, sex verification, and sample identity prediction using Omni2.5 M genotype [32]. Quality-controlled transcriptome data was available for 744 subjects at 49,385 probe level and was downloaded from http://adni.loni.usc.edu. Blood transcriptome data was available for 102 ADNI2 subjects with successfully processed diffusion procedure and year at which PaxGene sample was collected matching with scan year. Toll receptor signaling pathway was represented by 49 genes spanning 129 probes in the transcriptome data [31], and probe-level expressions were collapsed to gene-level using mean-max method [34]. The association between diffusion measures for each tract and gene expression was examined according to the following linear model: Diffusion measure $\alpha+$ Gene Expression + Sex + Age + RIN $+(1 \mid$ AffyPlate $)+\varepsilon$ where $\alpha$ is intercept, $\varepsilon$ is random error, and RIN is RNA integrity number. The aggregate effect of 49 Toll receptor signaling genes unto each diffusion measures was determined using sum of $\chi^{2}$ method [35, 36], and its significance was evaluated by 100,000 permutations $\left(P_{\text {permutation }}<0.05\right)$. 


\section{Statistical analysis and visualizations}

All statistical analyses were performed using Julia 1.0.3 (MIT, Cambridge, MA) [37]. The networks were visualized using spring-affinity algorithm. All heatmaps were drawn in $\mathrm{R}$ using Ward's method for hierarchical clustering (R Core Team, Vienna, Austria) [38].

\section{Results}

\section{Brain connectome by $\mathrm{dMRI}$ and associations with $\mathrm{AD}$ diagnosis}

The ADNI3 cohort ( $n=449$, the "Methods" section) [11], consisting of 347 healthy controls, 118 mild cognitive impaired (MCI), and $34 \mathrm{AD}$ patients, was interrogated for characterizing diffusion measures in 18 tracts derived using TRACULA [26] (Table 1). The brain volumes were positively correlated with $\mathrm{MD}$ and $\mathrm{RD}$ in all tracts (Additional file 1: Figure S4). RD was more significantly correlated with volume than MD. The average length of tracts was negatively correlated with MD and $\mathrm{RD}$ where MD was more correlated with tract average lengths than RD. In all 18 tracts of interest, $\mathrm{AxD}$ was positively correlated with FA and RD was positively correlated with MD.

The diffusion measures were compared against age, sex, years of education, marriage status, APOE4 genotype, and total brain volume (TBV) and disease diagnosis (Fig. 1a). Age was significantly associated with $\mathrm{AxD}, \mathrm{RD}$, and MD in all tracts $\left(P\right.$-values $\left.=2.2 \times 10^{-5} \sim 2.4 \times 10^{-17}\right)$ and with FA in a subset of tracts (16 out of 18 tracts below $P$-value $\left.<0.05, P_{\min }=4.2 \times 10^{-12}\right)$, consistent with reports in literature [39]. TBV was associated with RD, $\mathrm{MD}$, and FA in a number of tracts. Disease diagnosis status was associated with $\mathrm{AxD}, \mathrm{RD}$, and $\mathrm{MD}$ in a large number of tracts similar to findings in other studies [40]. After adjusting age, sex, and TBV effects, only disease diagnosis status remained significantly associated with the diffusion measures (Fig. 1b). Among all diffusion measures in 18 tracts, we identified 34 significant disease associations in a data-driven manner with $\mathrm{AxD}, \mathrm{RD}$, $\mathrm{MD}$, and FA in a number of tracts at $\mathrm{FDR}<0.05$ (Table 2). There was no AD diagnosis status association with bilateral CST and FMajor which are responsible for motor and visual functions.

The ADNI2 cohort ( $n=210$, the "Methods" section) [41], consisting of 75 healthy controls, $91 \mathrm{MCI}$, and 44 $\mathrm{AD}$ patients, is a cohort independent from the ADNI3 cohort (the "Methods" section). The same 18 tracts were derived using TRACULA [26]. The similar interrelationships among diffusion measures, covariates, and disease diagnosis were observed (Additional file 2: Table S7). After adjusting age, sex, and TBV effects, 11 diffusion measures were significantly associated with disease diagnosis at FDR $<0.05$ (Table 2, right) involving 4 of 18 tracts, bilateral CABs, bilateral SLFTs, L-SLFP, and LILF. Note that not only all the 11 associations overlapped with the 34 associations identified in ADNI3 cohort, but the direction of measure changes in response to diagnosis status was also replicated (Fig. 2). AxD, RD, and MD increased while FA decreased with disease diagnosis status (Fig. 2). The effect sizes and directions in ADNI2 and ADNI3 cohorts for the 34 associations identified in the ADNI3 cohort and the 11 replicated associations exhibited higher effect sizes than non-replicated associations (Fig. 3, Additional file 2: Table S8), suggesting a larger sample size is needed for replicating these associations of small effect sizes.

\section{Brain regions connected by different white matter tracts} We extracted path distribution information from each tract and calculated the probability of a tract reaching any Desikan-Killiany defined grey matter ROI [21]. Throughout this study, these measures were referred to as "reach probability." Reach probability was developed to allow focusing on only major tracts and integrative analysis between neuroimaging and transcriptome datasets. The reach probability was limited to white matter boundary neighboring each respective ROI in order to avoid amplifying the connection to ROI based on within-ROI streamline propagations in the tractography processes. Reach probability was derived for 18 tracts towards 82 ROIs, and 607 out of $1476(18 \times 82)$ probabilities had zero reach probability (41\%) (Additional file 2: Table S1). The non-zero reach probability followed an extreme value distribution (Additional file 1: Figure S2), and 203 ROI-tract pairs (33.4\%) were identified (the "Methods" section).

\section{Brain connectome by tissue-tissue transcriptional synchronization}

In order to investigate molecular connections between brain regions, we analyzed tissue-tissue co-regulation $[17,18]$ of transcriptomic data covering 17 post-mortem brain regions (Fig. 4) [12, 13]. Subjects shared for each pair of brain regions were in the range of $30-51$ subjects

Table 1 Demographic of ADNI2 and ADNI3

\begin{tabular}{|c|c|c|c|c|c|c|c|c|c|c|c|}
\hline & \multirow{2}{*}{$\begin{array}{l}\text { Age (years) } \\
\text { Mean } \pm \text { std }\end{array}$} & \multirow{2}{*}{$\begin{array}{l}\text { Gender } \\
\text { Males }\end{array}$} & \multicolumn{6}{|l|}{ Ethnicity } & \multicolumn{3}{|c|}{ Diagnosis } \\
\hline & & & Am. Indian/Alaskan & Asian & Black & White & More than one & Unknown & CN & $\mathrm{MCl}$ & Dementia \\
\hline ADNI3 $(n=499)$ & $73.8 \pm 8.4$ & $229(45.9 \%)$ & $1(0.2 \%)$ & $6(1.2 \%)$ & $15(3.0 \%)$ & 467 (93.6\%) & $8(1.6 \%)$ & $2(0.4 \%)$ & 347 & 118 & 34 \\
\hline ADNI2 $(n=210)$ & $73.5 \pm 6.8$ & 120 (57.1\%) & $1(0.5 \%)$ & $6(2.9 \%)$ & $8(3.8 \%)$ & 192 (91.4\%) & $3(1.4 \%)$ & $0(0.0 \%)$ & 75 & 91 & 44 \\
\hline
\end{tabular}




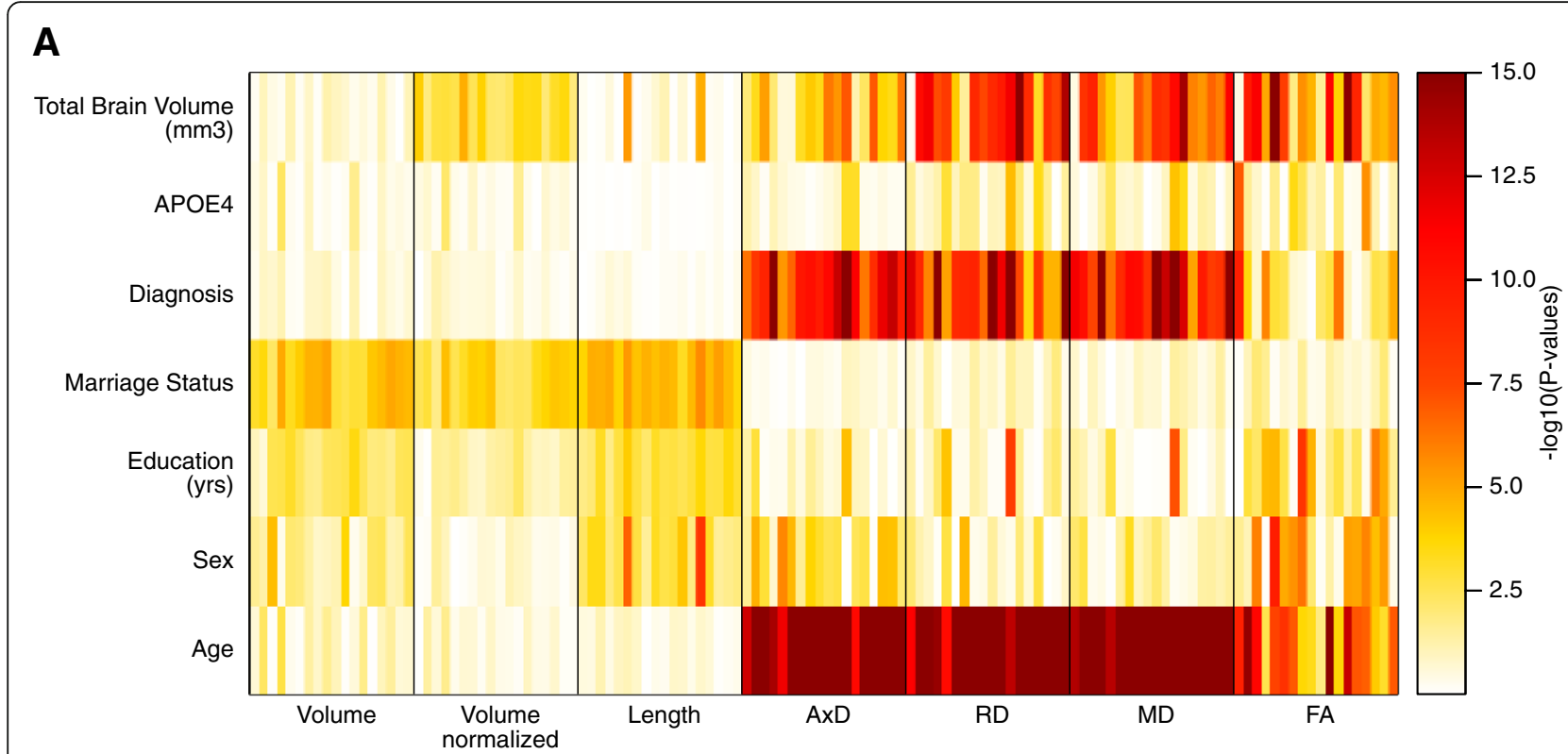

B

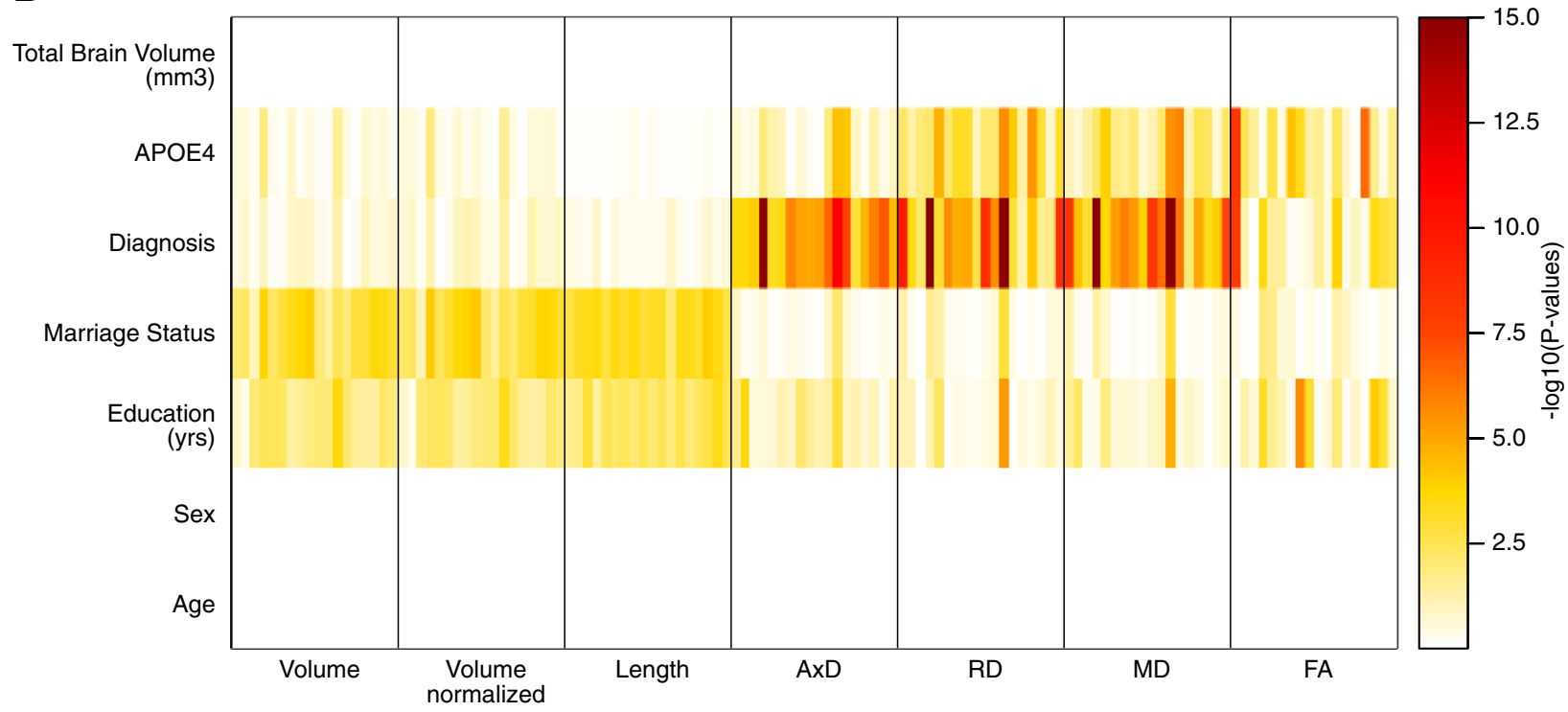

Fig. 1 Association between diffusion properties of 18 major tracts and covariates. a For 18 major tracts, covariates age, sex, years of education, marriage status, clinical diagnosis, APOE4 genotype, and total brain volume were tested against tract measures that are volume, volume normalized by total brain volume, tract length, axial diffusivity (AxD), radial diffusivity (RD), mean diffusivity (MD), and fractional anisotropy (FA). Rows of the heatmaps represent covariates. Individual association was - $\log 10$ transformed and displayed as heatmaps. Columns are organized by tract measure types separated by black vertical lines. For each tract measure type, individual tract associations are arranged in the following orders from left to right: forceps major, forceps minor, left anterior thalamic radiations (L-ATR), left cingulum—angular bundle (L-CAB), left cingulum - cingulate gyrus (L-CCG), left corticospinal tract (L-CST), left inferior longitudinal fasciculus (L-ILF), left superior longitudinal fasciculus parietal (L-SLFP), left superior longitudinal fasciculus temporal (L-SLFT), left uncinate fasciculus (L-UNC), right anterior thalamic radiations (R-ATR), right cingulum — angular bundle (R-CAB), right cingulum — cingulate gyrus (R-CCG), right corticospinal tract (R-CST), right inferior longitudinal fasciculus (R-ILF), right superior longitudinal fasciculus parietal (R-SLFP), right superior longitudinal fasciculus temporal (R-SLFT), right uncinate fasciculus (R-UNC). b Tract measures were adjusted for age, sex, and total brain volume before association testing, and only diagnosis associations remain strongly associated with diffusion-related measures $A x D, R D, M D$, and FA

depending on post-mortem tissue availability. There were $136(17 \times 16 / 2)$ possible brain region pairs among 17 brain regions. Brain connectome is defined by TTC of all gene pairs after adjusting Braak score in order to examine consistent gene synchronization between brain regions with regard to different disease diagnosis groups. TTCs were adjusted for covariates such as age, sex, post-mortem interval, $\mathrm{pH}$, and race [17] (detailed in the 
Table 2 Association between diffusion parameters and disease (FDR estimated independently)

\begin{tabular}{|c|c|c|c|c|c|c|c|c|c|c|c|c|}
\hline \multirow[b]{2}{*}{ Tract } & \multicolumn{6}{|c|}{ ADNI3 $(n=499)$} & \multicolumn{6}{|c|}{$\operatorname{ADNI2}(n=210)$} \\
\hline & Volume & Length & $A \times D$ & $\mathrm{RD}$ & $\mathrm{MD}$ & FA & Volume & Length & $A x D$ & $\mathrm{RD}$ & $\mathrm{MD}$ & FA \\
\hline FMajor & 0.41 & 0.83 & 0.55 & 0.09 & 0.14 & 0.02 & 0.89 & 0.18 & 0.64 & 0.07 & 0.07 & 0.08 \\
\hline Fminor & 0.41 & 0.85 & 0.06 & $4.1 \times 10^{-3}$ & 0.01 & 0.41 & 0.44 & 0.59 & 0.37 & 0.26 & 0.22 & 0.16 \\
\hline L-ATR & 0.73 & 0.86 & 0.04 & 0.16 & 0.08 & 0.90 & 0.30 & 0.81 & 0.63 & 0.54 & 0.52 & 0.65 \\
\hline$L-C A B^{* *}$ & 0.41 & 0.74 & $2.1 \times 10^{-5}$ & $1.9 \times 10^{-7}$ & $3.0 \times 10^{-7}$ & 0.04 & 0.01 & 0.10 & $2.7 \times 10^{-4 \dagger}$ & $9.3 \times 10^{-7 \dagger}$ & $9.2 \times 10^{-7 \dagger}$ & $0.05^{\dagger}$ \\
\hline L-CCG & 0.95 & 0.96 & 0.06 & 0.02 & 0.01 & 0.39 & 0.65 & 0.65 & 0.08 & 0.95 & 0.34 & 0.46 \\
\hline L-CST & 0.86 & 0.83 & 0.21 & 0.21 & 0.17 & 0.40 & 0.62 & 0.08 & 0.06 & 0.90 & 0.51 & 0.32 \\
\hline L-ILF** & 0.71 & 0.85 & 0.02 & 0.04 & 0.02 & 0.88 & 0.54 & 0.63 & $0.04^{+}$ & 0.19 & 0.05 & 0.65 \\
\hline L-SLFP** & 0.41 & 0.88 & 0.03 & 0.09 & 0.02 & 0.88 & 0.59 & 0.90 & $0.02^{+}$ & 0.67 & 0.22 & 0.30 \\
\hline L-SLFT** & 0.49 & 0.88 & 0.03 & 0.11 & 0.04 & 0.71 & 0.63 & 0.65 & $1.2 \times 10^{-3 \dagger}$ & 0.90 & 0.18 & 0.08 \\
\hline L-UNC & 0.85 & 0.85 & 0.03 & $1.1 \times 10^{-3}$ & $2.0 \times 10^{-3}$ & 0.55 & 0.08 & 0.63 & 0.18 & 0.11 & 0.08 & 0.76 \\
\hline R-ATR & 0.90 & 0.87 & 0.02 & 0.10 & 0.05 & 0.75 & 0.05 & 0.65 & 0.72 & 0.46 & 0.51 & 0.63 \\
\hline$R-C A B^{* *}$ & 0.60 & 0.63 & $4.8 \times 10^{-3}$ & $1.2 \times 10^{-5}$ & $2.3 \times 10^{-5}$ & 0.04 & 0.06 & 0.06 & $1.2 \times 10^{-3 \dagger}$ & $4.2 \times 10^{-3+}$ & $6.6 \times 10^{-4 \dagger}$ & 0.54 \\
\hline R-CCG & 0.93 & 0.85 & $4.8 \times 10^{-3}$ & 0.10 & 0.01 & 0.89 & 0.69 & 0.86 & 0.08 & 0.90 & 0.51 & 0.58 \\
\hline R-CST & 0.90 & 0.95 & 0.08 & 0.26 & 0.11 & 0.49 & 0.58 & 0.24 & 0.32 & 0.90 & 0.69 & 0.54 \\
\hline R-ILF & 0.34 & 0.83 & 0.04 & 0.07 & 0.04 & 0.95 & 0.95 & 0.95 & 0.09 & 0.86 & 0.46 & 0.95 \\
\hline R-SLFP & 0.75 & 0.67 & 0.02 & 0.41 & 0.10 & 0.06 & 0.65 & 0.65 & 0.07 & 0.89 & 0.51 & 0.38 \\
\hline R-SLFT** & 0.90 & 0.85 & 0.01 & 0.39 & 0.06 & 0.07 & 0.41 & 0.90 & $0.05^{\dagger}$ & 0.65 & 0.65 & 0.06 \\
\hline R-UNC & 0.75 & 0.85 & 0.04 & $1.6 \times 10^{-3}$ & $4.8 \times 10^{-3}$ & 0.20 & 0.13 & 0.79 & 0.54 & 0.86 & 0.65 & 0.76 \\
\hline
\end{tabular}

The associations below FDR $<0.05$ in each cohort study are presented in italics

**Tracts with associations identified in ADNI3 and replicated in ADNI2

${ }^{\dagger}$ Associations identified in ADNI3 and replicated in ADNI2

"Methods" section). The strength of brain region-region connections was measured by the number of significant TTC gene pairs. The distribution of significant TTC gene pair counts is shown in Fig. 5a for 136 region pairs, suggesting that only a fraction of brain regions were synchronized at the transcriptional level.

\section{Comparison of brain connectomes by diffusion MRI and genomics}

The post-mortem brain regions were originally labeled according to the Brodmann area map [12] and were matched to brain regions in Desikan-Killiany cortical atlas [21] that was used in the above tractography analysis (Additional file 2: Table S2). Among 136 possible brain region pairs, 64 region pairs were connected by white matter tracts defined by tractography. Among 64 tract-bound region pairs, 28 pairs were connected by $\mathrm{AD}$-associated tracts: L-CAB, L-ILF, L-SLFT, and L-SLFP. Among the top 10 percentile of region pairs $(n=14)$ containing the highest number of significant tissue-tissue correlated gene pairs, 10 were tract-bound (Fisher's exact test, $P=0.057$; Fig. 5b), and 7 out of top 10 percentile of region pairs $(n=14)$ were bound by AD-associated tracts (Fisher's exact test, $P=0.03$; Fig. $5 \mathrm{~b}$ ). This suggests that the brain connectomes defined by two different approaches overlap, especially for connections related to AD.

\section{Pathways associated with TTC gene pairs between different brain regions}

Different brain regions were connected by white matter tracts and synchronized at the transcriptional level as shown above. To investigate whether any biological pathways were transcriptionally synchronized between brain region pairs, we constructed bipartite clusters of TTC gene pairs for all 136 ROI pairs and identified gene modules for each brain region in ROI pairs (Fig. 4). Genes in the modules were annotated using PANTHER database [31], and pathways enriched among these genes at FDR $<0.05$ are listed in Additional file 2: Table S9. Among 169 pathways $\times$ 136 ROI pairs (22,984), 736 (3.2\%) pathways to ROI pair associations were significant, covering 83 pathways and 69 ROI pairs (Fig. 6a). A large fraction $(51 / 83=61.4 \%)$ of enriched pathways belonged to signaling pathways (Fisher exact test, $P=6.08 \times 10^{-9}$, Fig. 6a). The 69 ROI pairs were clustered to 3 clusters according to enriched pathways (Fig. 6a). The ROI pairs in cluster I (Fig. 6a), which were connected by multiple pathways, were enriched for tractbound ROI pairs and AD-associated tract-bound pairs $(P=$ 0.04 and 0.01 , respectively). This suggests that white matter tracts may serve as a mechanism of gene synchronization for signaling pathways, at least in brain regions present in ROI cluster I (PHG-TP, PHG-STG, ITG-MTG, ITG-PHG, IFC-ITG, PFC-STG, IFC-ITG). 


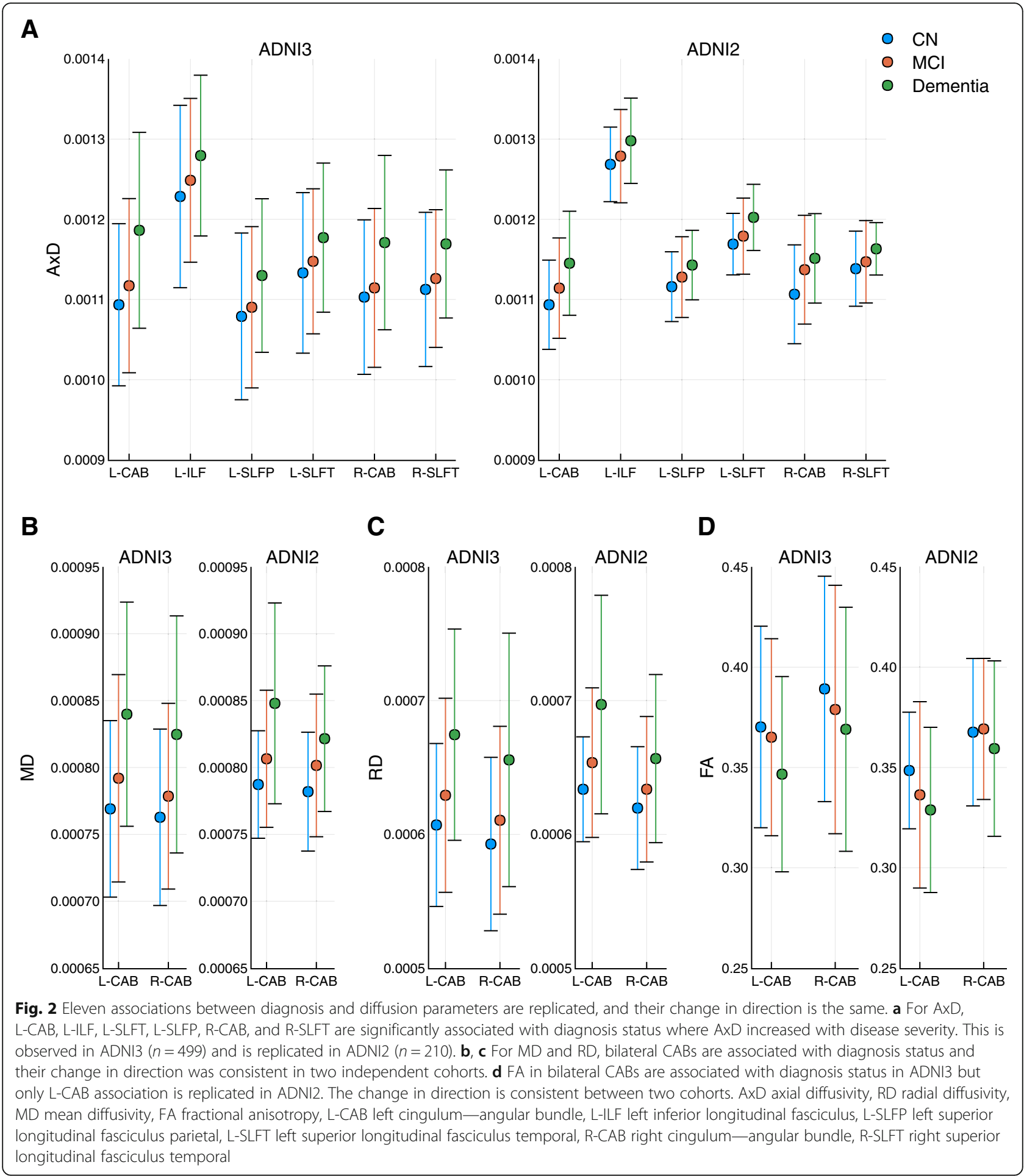

Next, we examined whether any pathways were preferentially involved in TTCs of tract-bound or ADassociated tract-bound ROI pairs (Fig. 6b, detailed in the "Methods" section). Apoptosis signaling pathway $(P=$ $0.006)$, EGF receptor signaling $(P=0.046)$, and metabotropic glutamate receptor (mGluR) I pathways $(P=$
0.049) were overrepresented in tract-bound region pairs (Additional file 2: Table S9). More pathways were preferentially involved in TTCs of AD-associated tract-bound ROI pairs, including apoptosis signaling $(P=0.008)$, muscarinic acetylcholine receptor (mAChR) 2 and 4 signaling pathway $(P=0.01)$, valine synthesis $(P=0.012)$, 
A

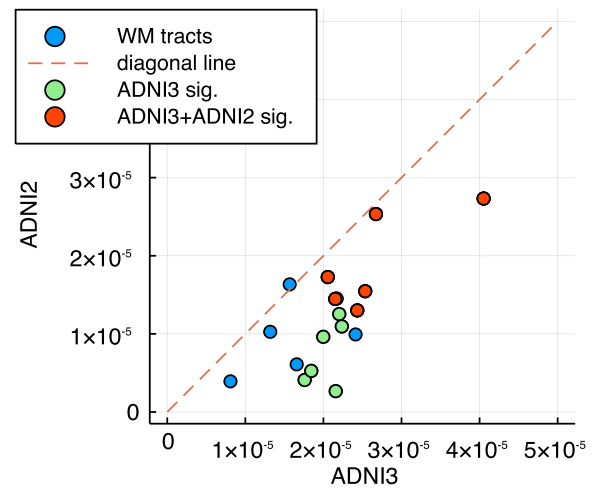

C

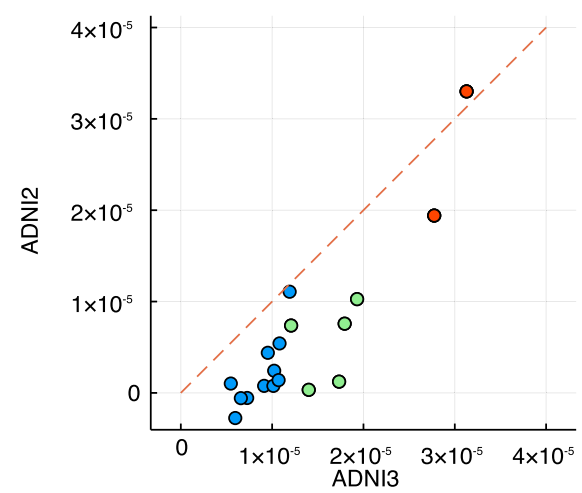

B

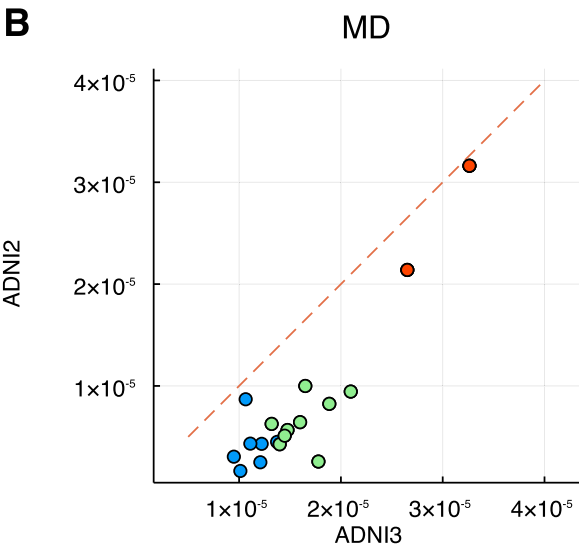

D

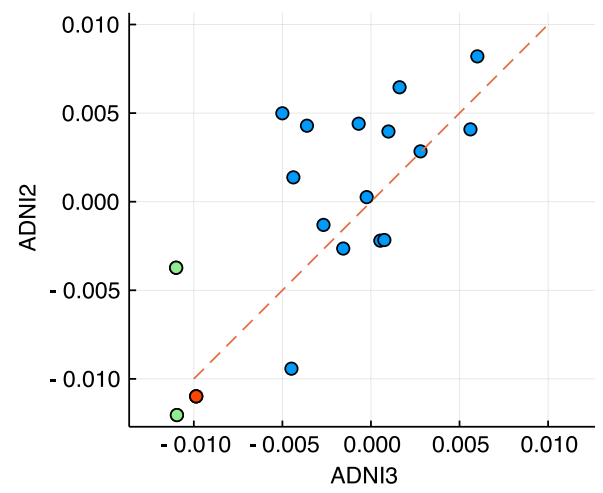

Fig. 3 The replicated associations had larger effect sizes than non-replicated associations. The 2D scatter plot of effect sizes in ADNI3 and ADNI2 suggests concordance of associations in the two studies. Red circles are associations replicated in ADNI2, and light-green circles represent nonreplicated associations. a-c The replicated associations in AxD, MD, and RD had larger effect sizes than non-replicated associations. $\mathbf{d} L-C A B$ association with FA had a large effect size relative to other comparisons and was replicated

PI3 kinase pathway $(P=0.013)$, endothelin signaling pathway $(P=0.016)$, histamine synthesis $(P=0.016)$, p38 MAPK pathway $(P=0.02)$, mGluR I pathway $(P=0.024)$, inflammation mediated by chemokine and cytokine signaling pathway $(P=0.028)$, mGluR II pathway $(P=$ 0.035), toll receptor signaling pathway $(P=0.045)$, adenine and hypoxanthine salvage pathway $(P=0.046)$ (Additional file 2: Table S9). The results suggest that signaling pathways, especially synaptic signaling and immune signaling pathways, involve in transcriptional synchronization between brain regions connected by white matter tracts.

\section{Toll receptor signaling pathway is overrepresented in both tract-bound and AD-associated tract-bound ROI pairs}

Gene modules derived from bipartite clustering TTC gene pairs were enriched in specific molecular pathways, mostly related to signaling. However, biological pathways in one region may not reciprocally synchronize the same pathway in another brain region because each region is accountable for their own distinct roles. For instance, our analyses show that $\mathrm{CN}$ and $\mathrm{ACC}$ were structurally connected (Additional file 2: Table S1) and literature supports that they are functionally connected [42]. However, $\mathrm{CN}$ and $\mathrm{ACC}$ are enriched in different neuron types (dopaminergic [43] and spindle neurons [44], respectively) and are responsible for different biological processes that may be mediated by differing molecular functions. Using 169 curated pathways as generalizable domains of molecular functions [31], we investigate how pathways are differentially interacting between brain regions. We defined pathway interactions specific to tractbound ROI pairs based on the chi-square test $(P<0.05$, Additional file 2: Table S5) and the same analysis was performed for $\mathrm{AD}$-associated tract-bound ROI pairs (Additional file 2: Table S6). Both pathway networks of tract-bound ROI pairs (G1) and AD-associated tractbound ROI pairs (G2) were made up of nodes that are signaling related (Fig. 7a, b). G2 had a larger number of pathway interactions than G1 (Fig. 7c), and the node with the most number of edges in G2 was toll receptor signaling pathway (Additional file 2: Table S10). The top two nodes with the highest number of edges in the G1 

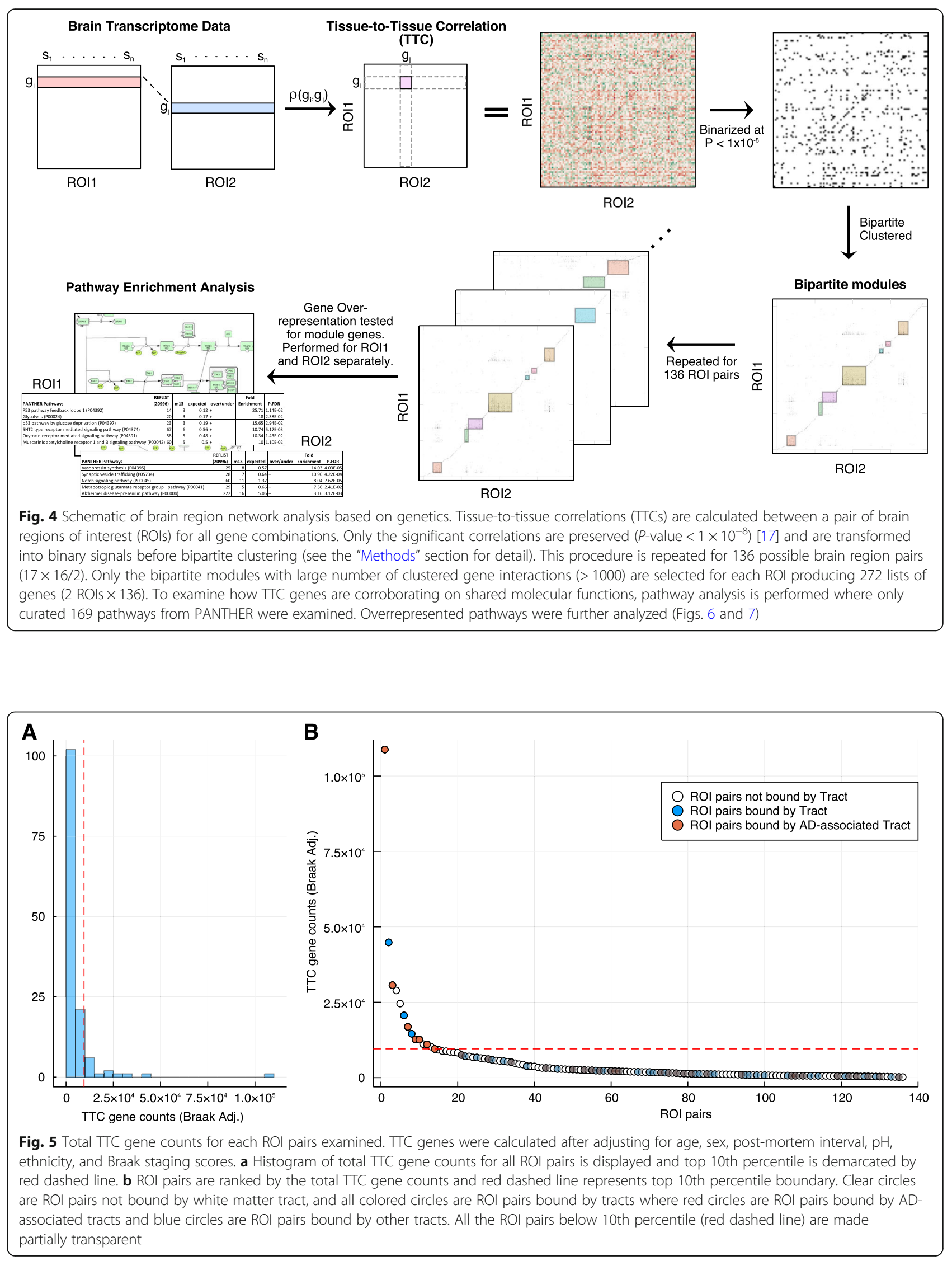


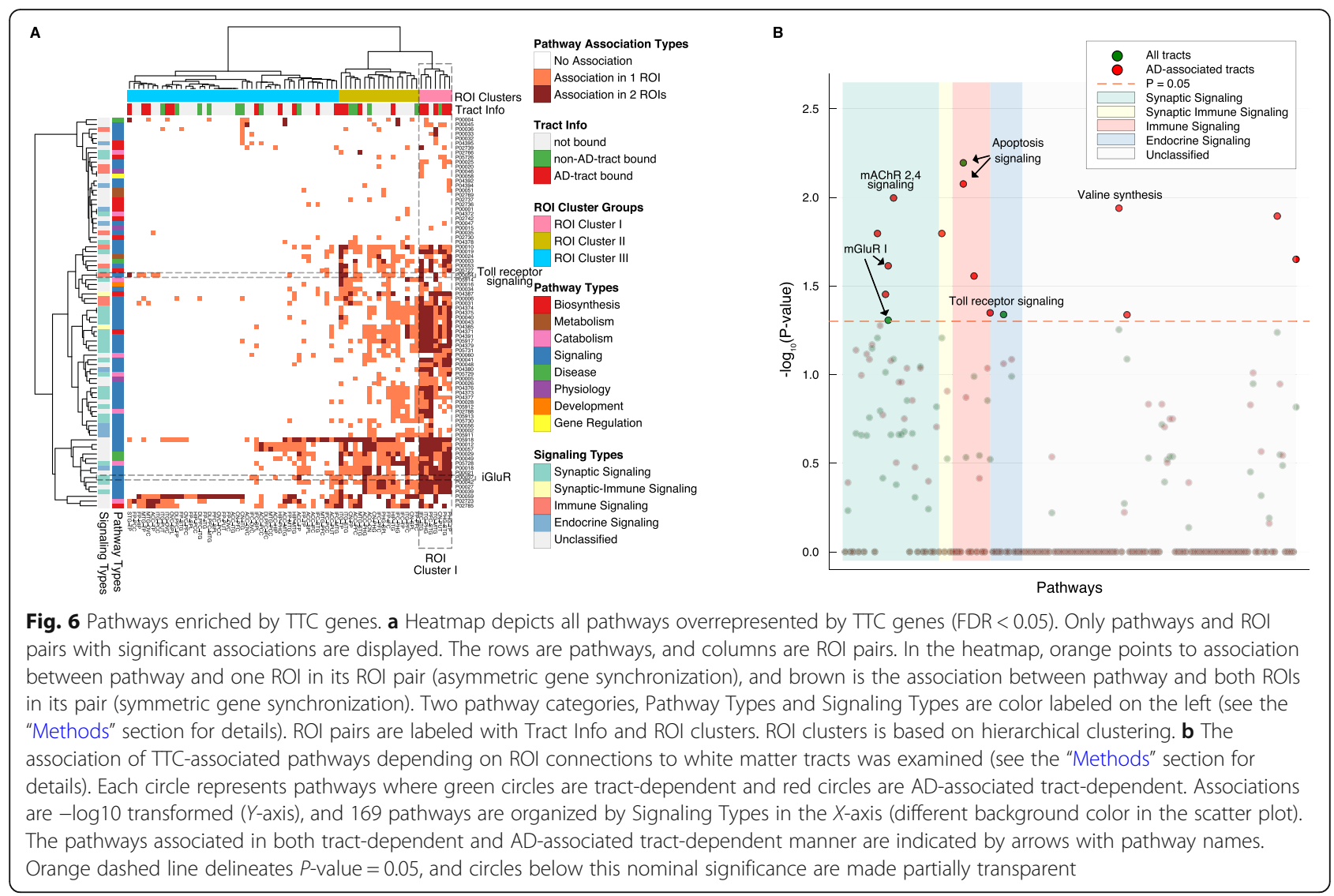

were ionotropic glutamate receptor (iGluR) pathway and toll receptor signaling pathway (Fig. 7c).

Alternatively, TTC genes in ROI pairs may be involved in shared molecular functions (symmetric synchronization). We examined pathways associated in both brain regions in a pair (Additional file 2: Table S11). For genes in TTC gene pairs of tract-bound ROI pairs, iGluR pathway $(P=0.021)$, toll receptor signaling pathway $(P=0.021)$, inflammation mediated by chemokine and cytokine signaling pathway $(P=0.047)$ were significantly overrepresented (Fig. $7 d)$. For genes in TTC gene pairs of AD-associated tract-bound region pairs, toll receptor signaling pathway $(P=0.003)$, iGluR pathway $(P=0.013)$, inflammation mediated by chemokine and cytokine signaling pathway $(P=0.031)$, PI3 kinase pathway $(P=0.031)$, mGluR group III pathway $(P=0.033)$, endothelin signaling pathway $(P=0.036)$, mGluR group II pathway $(P=0.036)$, mGluR group I pathway $(P=0.036), \mathrm{T}$ cell activation $(P=0.036), \quad 3$ adrenergic receptor signaling pathway $(P=0.036)$, and $\mathrm{mAChR} 1$ and 3 signaling pathway $(P=0.044)$ were preferentially involved (Fig. $7 \mathrm{~d})$.

\section{Toll receptor signaling genes in the blood associate with tract-wise diffusion measures in the brain}

Immune activities in the blood may reflect molecular states in the brain [45]. Because toll receptor signaling pathway was the most enriched pathway involved in symmetric synchronization between $\mathrm{AD}$-associated tract-bound ROI pairs (Fig. 7d), we interrogated how toll receptor signaling-related genes' expression in the blood associated with diffusion measures in the brain. There were 102 subjects with both blood expression data and dMRI scans in ADNI2 (the "Methods" section). We examined the pooled effect of 49 genes representing toll receptor signaling pathway [31] on diffusion measures of 18 tracts using sum of the chi-square method and compared them with the inferences based on 100,000 permutations $[35,36]$. Multiple diffusion measures including AxD of R-ATR $\left(P=1.0 \times 10^{-5}\right)$, R-CCG $(P=$ $\left.1.0 \times 10^{-5}\right)$, L-UNC $\left(P=5.6 \times 10^{-4}\right)$, L-CCG $(P=3.5 \times$ $\left.10^{-3}\right)$, L-ILF $\left(P=7.5 \times 10^{-3}\right)$, and R-SLFT $\left(P=7.6 \times 10^{-3}\right)$ were significantly associated with expression of genes in the toll receptor signaling pathway in the blood (Fig. 8a, Additional file 2: Table S12). RD (which measures diffusivity orthogonal to $\mathrm{AxD})$ of forceps minor $(P=5.6 \times$ $\left.10^{-3}\right)$ and L-CAB $\left(P=2.0 \times 10^{-2}\right)$ was significantly associated with the expression of toll receptor signalingrelated genes in the blood (Fig. 8c). MD which captures diffusivity in all directions was associated in forceps minor $\left(P=7.6 \times 10^{-3}\right)$, bilateral CCGs $\left(P=9.7 \times 10^{-3}\right.$ and $4.7 \times 10^{-3}$ left and right respectively), L-CAB $(P=3.4 \times$ 


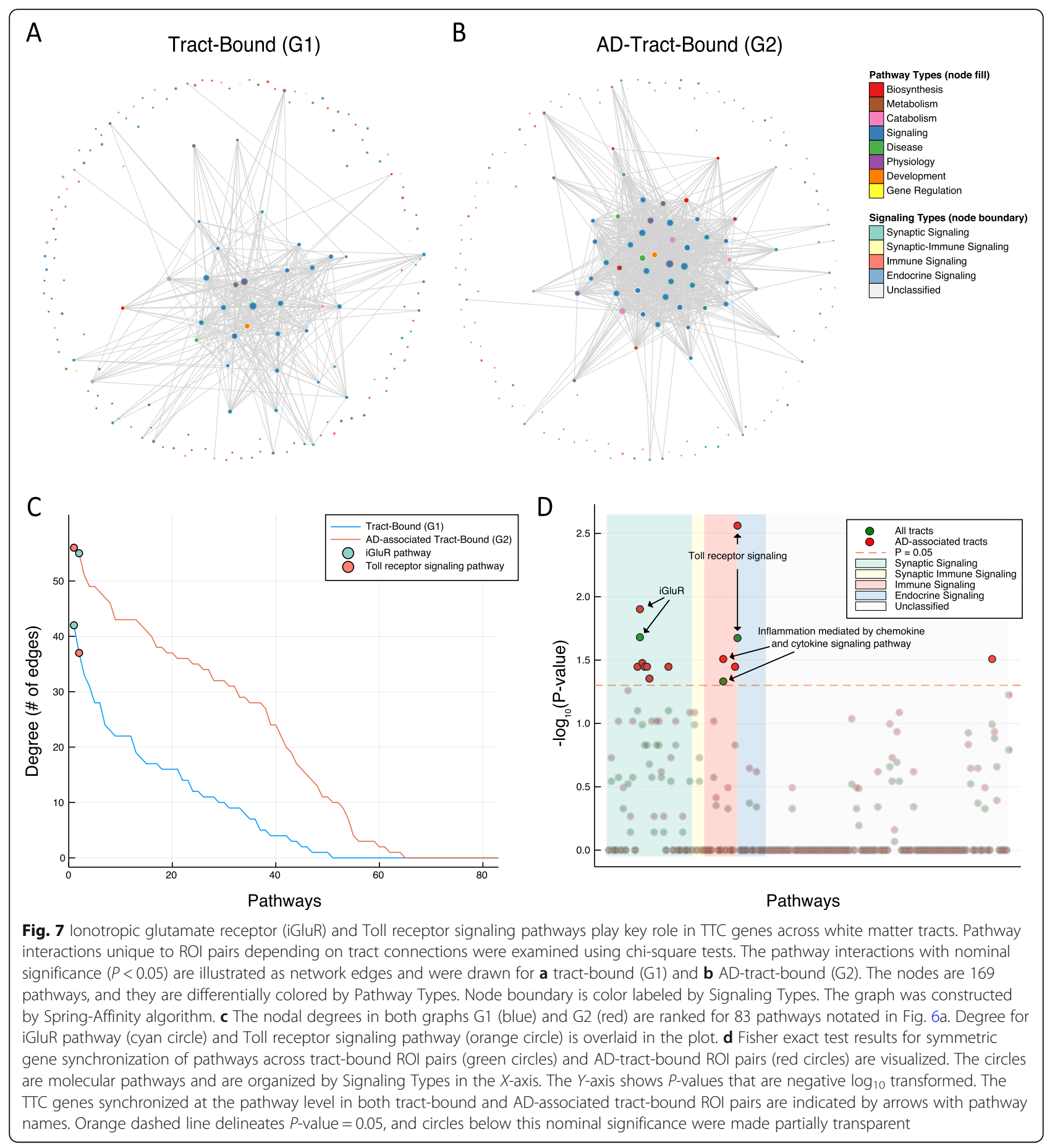

$\left.10^{-2}\right)$ L-SLFP $\left(P=1.5 \times 10^{-5}\right)$, and $\operatorname{L-SLFT}(P=2.7 \times$ $10^{-2}$ ) (Fig. 8b). FA which describes white matter integrity was associated in bilateral CABs $\left(P=1.0 \times 10^{-2}\right.$ and $4.9 \times 10^{-4}$ left and right respectively) and R-CCG $(P=$ $4.7 \times 10^{-2}$ ) (Fig. $8 \mathrm{~d}$ ). Similar to diagnosis associations (Table 2), we did not observe any toll receptor signaling gene expression association with bilateral CST and forceps major which are responsible for motor and visual functions.

\section{Discussion}

Tissues, organs and cell groups within organs, communicate with one another to perform biological functions in concert, and gene transcriptions are synchronized between tissues reflecting cross-tissue and cross-cell-group communications $[17,18]$. In the brain, white matter tracts serve as an important medium of brain regional cross-talk [46-48], and we observed that large numbers of genes were synchronized at the transcriptional level 

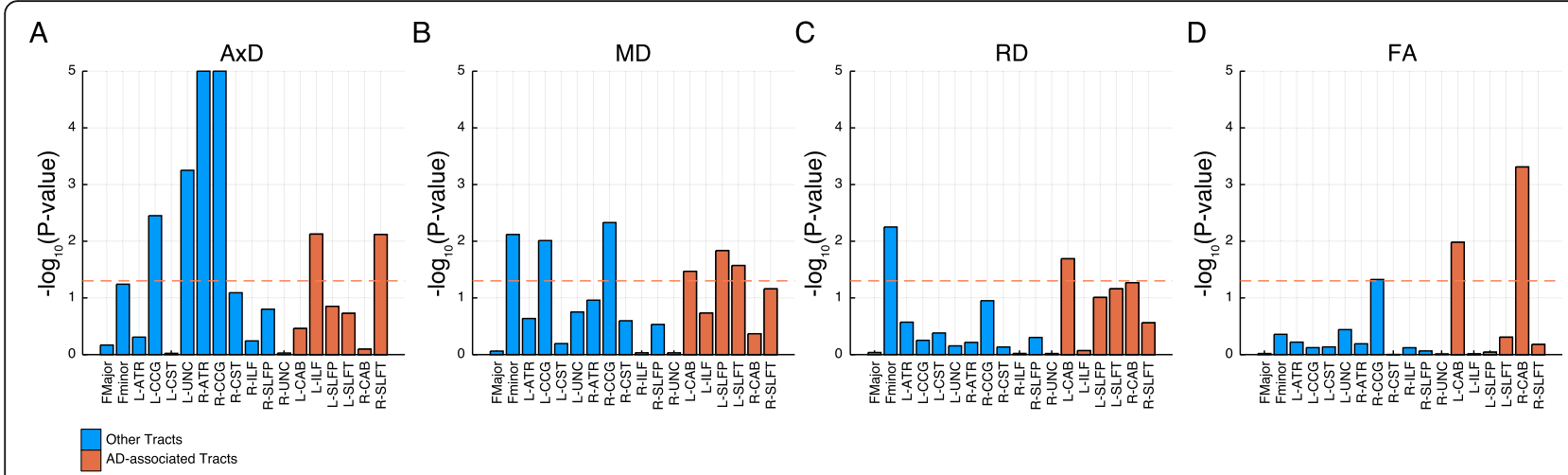

Fig. 8 Toll receptor signaling-related gene expression in the blood associate with diffusion properties in the white matter. Gene expression in the blood and dMRI scans in shared subjects were analyzed $(n=102)$. The transcriptome effects of toll receptor signaling pathway on a AxD, $\mathbf{b}$ MD, $\mathbf{c}$ $\mathrm{RD}$, and $\mathbf{d}$ FA in the 18 tracts were aggregated, and its en masse effect was approximated using 100,000x permutation. In all bar plots, ADassociated tracts replicated in two cohorts are in red, and other tracts are in blue. Orange dashed line represents $P$-value $=0.05$ threshold for significance

in tract-bound brain regions (Fig. 5b). Gene modules derived from bipartite clustering of TTC gene pairs between tract-bound brain regions were significantly overrepresented in signaling pathways (Fig. 6). Since axon bundles with synaptic connections constitute white matter tracts, identifying associations between mAChR, mGluR, and iGluR signaling pathways and TTC gene pairs were within our expectations (Fig. 6b). Toll receptor signaling pathway was the most enriched pathway in the symmetric gene synchronization between $\mathrm{AD}$ associated tract-bound brain regions (Fig. 7). There are at least two potential mechanisms: (1) Toll-like receptor (TLR) signaling plays a role in brain region-to-region communication via white matter tract and (2) TLR signaling pathways in brain regions and in the blood are synchronized [49]. The association between diffusion measures in major tracts and toll receptor signaling pathway activity in blood convolutes the two potential mechanisms. Although the mechanism is not clear, our results suggest the immune system's involvement in ADassociated brain region-to-region cross-talk.

TLRs play important roles in innate immunity in humans, and TLR activation in microglia due to neuropeptide aggregation is well established [50, 51]. However, the expression of TLRs is not limited to microglia [52, 53], but is also present in astrocytes [54], oligodendrocytes [55], neural progenitor cells [56, 57], and neurons [58]. The biology of TLRs is complex and goes beyond just recognizing pathogen-associated molecular patterns [59]. TLR3 can recognize double-stranded RNA for its activation [60], and the signaling cascade of TLRs varies for different neuronal cell types [61]. TLR2 and TLR4 are known to regulate hippocampal adult neurogenesis and neural progenitor cell differentiation [62]. TLR3 is associated with increased mature neurons in the hippocampus and enlarged dentate gyrus and the CA1 region [56].
TLR3 and TLR8 are present in the axonal tracts during the brain development and regulate neurite outgrowth and apoptosis [63-65]. In addition, differential expression of TLRs in human post-mortem brains are associated with alcohol addiction [66], depression [67, 68], and schizophrenia [69], and these neurological disorders are also associated with white matter abnormalities [70-72]. However, it is not known how TLRs may act on axonal degeneration and cross-communication between brain regions via axon fibers.

Diffusion-weighted imaging is a powerful tool in assessing microstructural changes of white matter in vivo, and diffusion parameters can capture white matter integrity [1]. In our work, TLR signaling expressions were associated with FA in bilateral CABs (Fig. 8). Because CABs have a strong connection to the hippocampus, white matter integrity measured by FA may be regulated by TLR signaling in the hippocampus and TLR-dependent adult neurogenesis [62]. AxD estimates parallel diffusivity along the direction of the highest diffusion and was significantly associated with expression of TLR signaling for bilateral-CCG, L-UNC, RATR, L-ILF, and R-SLFT. This suggests that TLR signaling may be involved in the loss of barriers restricting water diffusion in the associated tracts such as myelination level reduction or axon losses [73-75]. Although the association between diagnosis and diffusion measures in L-ILF and RSLFT was replicated in the ADNI2 cohort, L-UNC, R-ATR, and R-CCG findings failed to replicate in the ADNI2 cohort (Table 2). L-CCG was only nominally significant (FDR < 0.1 ) in both ADNI3 and ADNI2 cohorts (Table 2). This suggests that expression variation of genes in the TLR signaling pathway might be more powerful in detecting microscopic white matter abnormalities in comparison to diagnosis status, and further study may allow developing blood biomarkers relevant to disease-associated white matter changes in vivo. 
The sample size of ADNI3 was larger than the size of ADNI2 so that the ADNI3 study had a higher power to identify $\mathrm{AD}$ associations in diffusion imaging and not all associations were expected to be significant in the ADNI2. Besides the sample size, there were technical differences between the two cohorts [39, 76]. ADNI2 data was collected using older MR pulse sequence and was captured at $2.7-\mathrm{mm}^{3}$ resolution. ADNI3 adopted the optimized protocol established by Human Connectome Project as the standard across multiple centers and gained higher resolution at $2.0 \mathrm{~mm}^{3}$ [11]. There were 16 and 50 research sites involved in ADNI2 and ADNI3 studies, respectively. Four hundred nine out of $499 \mathrm{im}-$ ages in the ADNI3 dataset were acquired from 37 research sites that were not included in the ADNI2 (Additional file 2: Table S13). The results from the multi-center studies are unlikely due to biases from a few sites. As noted in the "Methods" section, we included only imaging data of participants that were unique to ADNI3 as the ADNI3 cohort so that there was no overlap between the ADNI2 and ADNI3 cohorts in our analyses. The identified imaging-based disease associations were also consistent with known findings [77, 78]. All these results together suggest that the associations between neuroimaging features and $\mathrm{AD}$ are robust to the differences between ADNI3 and ADNI2. Additionally, the replicated associations had larger effect size than the non-replicated ones, suggesting associations of smaller effect sizes require a larger sample size to validate.

There are limitations in our analyses and ADNI studies in general. Majority of the participants in the ADNI2 and ADNI3 studies were white $(91.4 \%$ and $93.6 \%$, respectively). Even though some common associations between neuroimaging features and $\mathrm{AD}$ were identified in ADNI2 and ADNI3 cohorts, whether the associations hold in other ethnic groups needs further studies. Additionally, there were only 17 brain regions available to construct transcriptome-based brain connectome. The limited spatial resolution of this work may increase false negatives. The Allen Human Brain Atlas has more complete coverage of the brain spatially [79], but is limited to only 6 individuals whereas we conducted our study using 30-51 subjects depending on the brain region. Although spatially limited, our work is much better powered than the Allen Human Brain Atlas in examining correlated expression between brain regions and should better reflect the population information. Another limitation is that our study only examined gene synchronization by major white matter tracts whereas gene synchronization between two brain regions may be mediated through multiple mechanisms, including (1) direct neighbor (cis), (2) WM connected (trans), and (3) functionally connected (multi). Future works are needed to address these different gene synchronization models.

\section{Conclusion}

Overall, this is the first study that investigates brain connectomes of white matter tracts and gene synchronization in human brains. For this, we developed a method that directly examines the enrichment of TTC genes in tract-bound brain regions and further performed molecular network analysis based on tract-wise connection information. Despite various limitations, we report that TTCs of genes in signaling pathways were significantly associated with brain regional cross-talk through white matter tracts. We further report that iGluR and toll receptor signaling pathways play a pivotal role in region-to-region communication and synaptic and immune interplay between brain regions may posit novel insights towards AD etiology.

\section{Supplementary information}

Supplementary information accompanies this paper at https://doi.org/10. 1186/s12916-019-1488-1.

\section{Additional file 1: Supplementary figures.}

Additional file 2: Table S1. Probability of tract reaching brain region (Reach probability). Table S2. Brain region mapping between DesikanKillany atlas and post-mortem brain labels. Table S3. Number of Tissueto-Tissue (TTC) correlated genes for each pair of Region-of-interests (ROIs). Table S4. Pathway list with annotated subtypes. Table S5. Pathway interactions in brain region pairs that are that are significantly different in tract-bound. Table S6. Pathway interactions in brain region pairs that are that are significantly different in AD-tract-bound. Table S7. Association between covariates and diffusion measures in each tract. Table S8. Effect sizes for associations in ADNI3 and ADNI2. Table S9. Pathway overrepresentation analysis between brain region pairs connected by white matter tracts and region pairs not connected by tracts. Table S10. Pathway interaction graph (degree). Table S11. Pathway overrepresentation analysis of symmetric gene synchronization in brain region pairs connected by white matter tracts. Table S12. Association between gene expression of Toll receptor signaling in the blood and diffusion measures in the brain. Table S13. Number of subjects in each study per site.

\section{Abbreviations}

ACC: Anterior cingulate cortex; AD: Alzheimer's disease; ADNI: Alzheimer's Disease Neuroimaging Initiative; AxD: Axial diffusivity; CN: Caudate nucleus; DLPFC: Dorsolateral prefrontal cortex; dMRI: Diffusion MRI; FA: Fractional anisotropy; FDR: False discovery rate; FMajor: Forceps major; Fminor: Forceps minor; FP: Frontal pole; HIP: Hippocampus; IFC: Inferior frontal cortex;

iGluR: Ionotropic glutamate receptor; ITG: Inferior temporal gyrus; L-ATR: Left anterior thalamic; L-CAB: Left cingulum - angular bundle; L-CCG: Left cingulum—cingulate gyrus; L-CST: Left corticospinal tract; L-ILF: Left inferior longitudinal fasciculus; L-SLFP: Left superior longitudinal fasciculus parietal; L-SLFT: Left superior longitudinal fasciculus temporal; L-UNC: Left uncinate fasciculus; mAChR: Muscarinic acetylcholine receptor; MCl: Mild cognitive impaired; MD: Mean diffusivity; mGluR: Metabotropic glutamate receptor; MRI: Magnetic resonance imaging; MTG: Middle temporal gyrus;

OVC: Occipital visual cortex; PANTHER: Protein Analysis Through Evolutionary Relationships; PCC: Posterior cingulate cortex; PCG: Precentral gyrus; PFC: Prefrontal cortex; PHG: Parahippocampal gyrus; PUT: Putamen; QC: Quality check; R-ATR: Right anterior thalamic radiations; R-CAB: Right cingulum — angular bundle; R-CCG: Right cingulum — cingulate gyrus; R-CST: Right corticospinal tract; RD: Radial diffusivity; R-ILF: Right inferior longitudinal fasciculus; ROI: Region of interest; R-SLFP: Right superior longitudinal fasciculus parietal; R-SLFT: Right superior longitudinal fasciculus temporal; R-UNC: Right uncinate fasciculus; SPL: Superior parietal lobule; STG: Superior temporal gyrus; TBV: Total brain volume; TLRs: Toll-like 
receptors; TP: Temporal pole; TRACULA: TRActs Constrained by UnderLying Anatomy; TTCs: Tissue-to-tissue correlations

\section{Acknowledgements}

Data collection and sharing for this project was funded by the Alzheimer's Disease Neuroimaging Initiative (ADNI) (National Institutes of Health Grant U01 AG024904) and DOD ADNI (Department of Defense award number W81XWH-12-2-0012). ADNI is funded by the National Institute on Aging, the National Institute of Biomedical Imaging and Bioengineering, and through generous contributions from the following: AbbVie, Alzheimer's Association; Alzheimer's Drug Discovery Foundation; Araclon Biotech; BioClinica, Inc.; Biogen; Bristol-Myers Squibb Company; CereSpir, Inc.; Cogstate; Eisai Inc.; Elan Pharmaceuticals, Inc.; Eli Lilly and Company; Eurolmmun; F. Hoffmann-La Roche Ltd. and its affiliated company Genentech, Inc.; Fujirebio; GE Healthcare; IXICO Ltd.; Janssen Alzheimer Immunotherapy Research \& Development, LLC.; Johnson \& Johnson Pharmaceutical Research \& Development LLC.; Lumosity; Lundbeck; Merck \& Co., Inc.; Meso Scale Diagnostics, LLC.; NeuroRx Research; Neurotrack Technologies; Novartis Pharmaceuticals Corporation; Pfizer Inc.; Piramal Imaging; Servier; Takeda Pharmaceutical Company; and Transition Therapeutics. The Canadian Institutes of Health Research is providing funds to support ADNI clinical sites in Canada. Private sector contributions are facilitated by the Foundation for the National Institutes of Health (www.fnih.org). The grantee organization is the Northern California Institute for Research and Education, and the study is coordinated by the Alzheimer's Therapeutic Research Institute at the University of Southern California. ADNI data are disseminated by the Laboratory for Neuro Imaging at the University of Southern California.

\section{Declarations}

This is not a human study. All data used in the study are from public databases. No new data is generated in the study.

\section{Authors' contributions}

YJW and JZ conceived and designed the study. VH, PK, and RP contributed to the sample collection and microarray experiments. ADNI provided neuroimaging data, relevant clinical data, and blood transcriptome data. YJW and JZ contributed to the data analysis and its interpretation. YJW and JZ wrote and edited the manuscript. All authors contributed to the result interpretation and discussion. All authors read and approved the final manuscript.

\section{Funding}

The study is partially supported by NIH AG046170 (VH, SG, EES, JZ), HG008451 (JZ), and AG024904 (ADNI).

\section{Availability of data and materials}

ADNI is available at http://adni.loni.usc.edu. Post-mortem transcriptome data is available at GSE84422.

\section{Competing interests}

The authors declare that they have no competing interests.

\section{Author details}

'Department of Genetics and Genomic Sciences, Icahn School of Medicine at Mount Sinai, New York, NY 10029, USA. ${ }^{2}$ Department of Psychiatry, Icahn School of Medicine at Mount Sinai, New York, NY 10029, USA. ${ }^{3}$ Department of Neuroscience, Icahn School of Medicine at Mount Sinai, New York, NY 10029, USA. ${ }^{4}$ Department of Neurology, Icahn School of Medicine at Mount Sinai, New York, NY 10029, USA. ${ }^{5}$ Sema4, Stamford, CT 06902, USA.

\section{Received: 3 July 2019 Accepted: 24 December 2019}

\section{Published online: 06 February 2020}

\section{References}

1. Shi $Y$, Toga AW. Connectome imaging for mapping human brain pathways. Mol Psychiatry. 2017;22:1230-40. https://doi.org/10.1038/mp.2017.92.

2. Smith SM, Beckmann CF, Andersson J, Auerbach EJ, Bijsterbosch J, Douaud $\mathrm{G}$, et al. Resting-state fMRI in the Human Connectome Project. Neuroimage. 2013;80:144-68.

3. Filippi M, Basaia S, Canu E, Imperiale F, Magnani G, Falautano M, et al. Changes in functional and structural brain connectome along the
Alzheimer's disease continuum. Mol Psychiatry. 2018. https://doi.org/10. 1038/s41380-018-0067-8

4. Brier MR, Thomas JB, Ances BM. Network dysfunction in Alzheimer's disease: refining the disconnection hypothesis. Brain Connect. 2014;4:299-311. https://doi.org/10.1089/brain.2014.0236.

5. Sporns O. Contributions and challenges for network models in cognitive neuroscience. Nat Neurosci. 2014;17:652-60. https://doi.org/10.1038/nn.3690.

6. Wolf L, Goldberg C, Manor N, Sharan R, Ruppin E. Gene expression in the rodent brain is associated with its regional connectivity. PLoS Comput Biol. 2011;7.

7. French $L$, Pavlidis P. Relationships between gene expression and brain wiring in the adult rodent brain. PLoS Comput Biol. 2011;7:e1001049. https://doi.org/10.1371/journal.pcbi.1001049.

8. Richiardi J, Altmann A, Jonas R. Correlated gene expression supports synchronous activity in brain networks. Science (80- ). 2015;348:11-4. https://doi.org/10.1126/science.1255905

9. Zhang B, Gaiteri C, Bodea LG, Wang Z, McElwee J, Podtelezhnikov AA, et al. Integrated systems approach identifies genetic nodes and networks in lateonset Alzheimer's disease. Cell. 2013;153:707-20. https://doi.org/10.1016/j. cell.2013.03.030

10. Raj T, Li YI, Wong G, Humphrey J, Wang M, Ramdhani S, et al. Integrative transcriptome analyses of the aging brain implicate altered splicing in Alzheimer's disease susceptibility. Nat Genet. 2018;50:1584-92. https://doi. org/10.1038/s41588-018-0238-1.

11. Weiner MW, Veitch DP, Aisen PS, Beckett LA, Cairns NJ, Green RC, et al. The Alzheimer's Disease Neuroimaging Initiative 3: continued innovation for clinical trial improvement. Alzheimer's Dementia. 2017;13:561-71. https:// doi.org/10.1016/j.jalz.2016.10.006

12. Haroutunian $V$, Katsel $P$, Schmeidler J. Transcriptional vulnerability of brain regions in Alzheimer's disease and dementia. Neurobiol Aging. 2009;30:56173. https://doi.org/10.1016/j.neurobiolaging.2007.07.021.

13. Wang M, Roussos P, McKenzie A, Zhou X, Kajiwara Y, Brennand KJ, et al. Integrative network analysis of nineteen brain regions identifies molecular signatures and networks underlying selective regional vulnerability to Alzheimer's disease. Genome Med. 2016;8:104. https://doi.org/10.1186/ s13073-016-0355-3.

14. Woo YJ, Wang T, Guadalupe T, Nebel RA, Vino A, Del Bene VA, et al. A common CYFIP1 variant at the 15q11.2 disease locus is associated with structural variation at the language-related left supramarginal gyrus. PLoS One. 2016;11:e0158036. https://doi.org/10.1371/journal.pone.0158036.

15. Stein JL, Medland SE, Vasquez AA, Hibar DP, Senstad RE, Winkler AM, et al. Identification of common variants associated with human hippocampal and intracranial volumes. Nat Genet. 2012;44. https://doi.org/10.1038/ng.2250.

16. Munafò MR, Nosek BA, Bishop DVM, Button KS, Chambers CD, Percie Du Sert N, et al. A manifesto for reproducible science. Nat Hum Behav. 2017;1: 1-9. https://doi.org/10.1038/s41562-016-0021.

17. Dobrin R, Zhu J, Molony C, Argman C, Parrish ML, Carlson S, et al. Multi-tissue coexpression networks reveal unexpected subnetworks associated with disease. Genome Biol. 2009;10:R55. https://doi.org/10.1186/gb-2009-10-5-r55.

18. Long Q, Argmann C, Houten SM, Huang T, Peng S, Zhao Y, et al. Inter-tissue coexpression network analysis reveals DPP4 as an important gene in heart to blood communication. Genome Med. 2016;8:15. https://doi.org/10.1186/ s13073-016-0268-1.

19. Li X, Morgan PS, Ashburner J, Smith J, Rorden C. The first step for neuroimaging data analysis: DICOM to NIfTI conversion. J Neurosci Methods. 2016;264:47-56. https://doi.org/10.1016/j.jneumeth.2016.03.001.

20. Fischl B, van der Kouwe A, Destrieux C, Halgren E, Ségonne F, Salat DH, et al. Automatically parcellating the human cerebral cortex. Cereb Cortex. 2004;14:11-22. https://doi.org/10.1093/cercor/bhg087.

21. Desikan RS, Ségonne F, Fischl B, Quinn BT, Dickerson BC, Blacker D, et al. An automated labeling system for subdividing the human cerebral cortex on MRI scans into gyral based regions of interest. Neuroimage. 2006;31:968-80. https://doi.org/10.1016/j.neuroimage.2006.01.021.

22. Jenkinson M, Beckmann CF, Behrens TEJ, Woolrich MW, Smith SM. FSL. Neuroimage. 2012;62:782-90. https://doi.org/10.1016/j.neuroimage.2011. 09.015.

23. Behrens TEJ, Berg HJ, Jbabdi S, Rushworth MFS, Woolrich MW. Probabilistic diffusion tractography with multiple fibre orientations: what can we gain? Neuroimage. 2007;34:144-55. https://doi.org/10.1016/j.neuroimage.2006.09.018.

24. Smith SM, Jenkinson M, Woolrich MW, Beckmann CF, Behrens TEJ, Johansen-Berg $\mathrm{H}$, et al. Advances in functional and structural MR image 
analysis and implementation as FSL. Neuroimage. 2004;23(Suppl 1):S208-19. https://doi.org/10.1016/..neuroimage.2004.07.051.

25. Behrens TEJ, Johansen-Berg H, Woolrich MW, Smith SM, Wheeler-Kingshott CAM, Boulby PA, et al. Non-invasive mapping of connections between human thalamus and cortex using diffusion imaging. Nat Neurosci. 2003;6: 750-7. https://doi.org/10.1038/nn1075.

26. Yendiki A, Panneck P, Srinivasan P, Stevens A, Zöllei L, Augustinack J, et al. Automated probabilistic reconstruction of white-matter pathways in health and disease using an atlas of the underlying anatomy. Front Neuroinform. 2011;:23. doi:https://doi.org/10.3389/fninf.2011.00023.

27. Barber MJ. Modularity and community detection in bipartite networks. Phys Rev E Stat Nonlin Soft Matter Phys. 2007;76 6 Pt 2:066102. doi:https://doi. org/10.1103/PhysRevE.76.066102.

28. Liu X, Murata T. An efficient algorithm for optimizing bipartite modularity in bipartite networks. J Adv Comput Intell Intell Informatics. 2010;14:408-15. https://doi.org/10.20965/jaciii.2010.p0408.

29. Costa A, Hansen P. A locally optimal hierarchical divisive heuristic for bipartite modularity maximization. Optim Lett. 2014;8:903-17. https://doi. org/10.1007/s11590-013-0621-x.

30. Beckett SJ. Improved community detection in weighted bipartite networks subject category : subject areas : author for correspondence : R Soc open Sci; 2016.

31. Mi H, Muruganujan A, Huang X, Ebert D, Mills C, Guo X, et al. Protocol update for large-scale genome and gene function analysis with the PANTHER classification system (v.14.0). Nat Protoc. 2019;14:703-21. https:// doi.org/10.1038/s41596-019-0128-8.

32. Saykin AJ, Shen L, Yao X, Kim S, Nho K, Risacher SL, et al. Genetic studies of quantitative $\mathrm{MCl}$ and $\mathrm{AD}$ phenotypes in ADNl: progress, opportunities, and plans. Alzheimers Dement. 2015;11:792-814. https://doi.org/10.1016/j.jalz. 2015.05.009.

33. Irizarry RA, Hobbs B, Collin F, Beazer-Barclay YD, Antonellis K, Scherf U, et al. Exploration, normalization, and summaries of high density oligonucleotide array probe level data. Biostatistics. 2003;4:249-64. https://doi.org/10.1093/ biostatistics/4.2.249.

34. Miller JA, Cai C, Langfelder P, Geschwind DH, Kurian SM, Salomon DR, et al. Strategies for aggregating gene expression data: the collapseRows $R$ function. BMC Bioinformatics. 2011;12:322. https://doi.org/10.1186/14712105-12-322.

35. Liu JZ, McRae AF, Nyholt DR, Medland SE, Wray NR, Brown KM, et al. A versatile gene-based test for genome-wide association studies. Am J Hum Genet. 2010;87:139-45. https://doi.org/10.1016/j.ajhg.2010.06.009.

36. Wang T, Zhou B, Guo T, Bidlingmaier M, Wallaschofski H, Teumer A, et al. A robust method for genome-wide association meta-analysis with the application to circulating insulin-like growth factor I concentrations. Genet Epidemiol. 2014;38:162-71. https://doi.org/10.1002/gepi.21766.

37. Bezanson J, Edelman A, Karpinski S, Shah VB. Julia: a fresh approach to numerical computing. SIAM Rev. 2017;59:65-98. https://doi.org/10.1137/ 141000671.

38. R Core Team. R: a language and environment for statistical computing. 2019. https://www.r-project.org/.

39. Zavaliangos-Petropulu A, Nir TM, Thomopoulos SI, Reid RI, Bernstein MA Borowski B, et al. Diffusion MRI indices and their relation to cognitive impairment in brain aging: the updated multi-protocol approach in ADNI3. Front Neuroinform. 2019;13 February:2. doi:https://doi.org/10.3389/fninf. 2019.00002.

40. Araque Caballero MÁ, Suárez-Calvet M, Duering M, Franzmeier N, Benzinger $\mathrm{T}$, Fagan $\mathrm{AM}$, et al. White matter diffusion alterations precede symptom onset in autosomal dominant Alzheimer's disease. Brain. 2018;141:3065-80. https://doi.org/10.1093/brain/awy229.

41. Weiner MW, Aisen PS, Jack CR, Jagust WJ, Trojanowski JQ, Shaw L, et al. The Alzheimer's disease neuroimaging initiative: progress report and future plans. Alzheimers Dement. 2010;6:202-11.e7. https://doi.org/10.1016/j.jalz. 2010.03.007.

42. Robinson JL, Laird AR, Glahn DC, Blangero J, Sanghera MK, Pessoa L, et al. The functional connectivity of the human caudate: an application of metaanalytic connectivity modeling with behavioral filtering. Neuroimage. 2012; 60:117-29. https://doi.org/10.1016/j.neuroimage.2011.12.010.

43. Nakamura K, Hikosaka O. Role of dopamine in the primate caudate nucleus in reward modulation of saccades. J Neurosci. 2006;26:5360-9. https://doi. org/10.1523/JNEUROSCl.4853-05.2006.
44. Nimchinsky EA, Vogt BA, Morrison JH, Hof PR. Spindle neurons of the human anterior cingulate cortex. J Comp Neurol. 1995;355:27-37. https:// doi.org/10.1002/cne.903550106.

45. Sankowski R, Mader S, Valdés-Ferrer SI. Systemic inflammation and the brain: novel roles of genetic, molecular, and environmental cues as drivers of neurodegeneration. Front Cell Neurosci. 2015;28. doi:https://doi.org/10.3389/ fncel.2015.00028.

46. Honey CJ, Sporns O, Cammoun L, Gigandet X, Thiran JP, Meuli R, et al. Predicting human resting-state functional connectivity from structural connectivity. Proc Natl Acad Sci U S A. 2009;106:2035-40. https://doi.org/10. 1073/pnas.0811168106.

47. van den Heuvel MP, Mandl RCW, Kahn RS, Hulshoff Pol HE. Functionally linked resting-state networks reflect the underlying structural connectivity architecture of the human brain. Hum Brain Mapp. 2009;30:3127-41. https:// doi.org/10.1002/hbm.20737.

48. Peer M, Nitzan M, Bick AS, Levin N, Arzy S. Evidence for functional networks within the human brain's white matter. J Neurosci. 2017;37:6394-407. https://doi.org/10.1523/JNEUROSCI.3872-16.2017.

49. Stolp HB, Liddelow SA, Sá-Pereira I, Dziegielewska KM, Saunders NR. Immune responses at brain barriers and implications for brain development and neurological function in later life. Front Integr Neurosci. 2013;1-14.

50. Letiembre M, Liu Y, Walter S, Hao W, Pfander T, Wrede A, et al. Screening of innate immune receptors in neurodegenerative diseases: a similar pattern. Neurobiol Aging. 2009;30:759-68. https://doi.org/10.1016/j.neurobiolaging. 2007.08.018.

51. Liu Y, Walter S, Stagi M, Cherny D, Letiembre M, Schulz-Schaeffer W, et al. LPS receptor (CD14): a receptor for phagocytosis of Alzheimer's amyloid peptide. Brain. 2005;128(Pt 8):1778-89. https://doi.org/10.1093/brain/ awh531.

52. Olson JK, Miller SD. Microglia initiate central nervous system innate and adaptive immune responses through multiple TLRs. J Immunol. 2004;173: 3916-24. https://doi.org/10.4049/jimmunol.173.6.3916.

53. Galatro TF, Holtman IR, Lerario AM, Vainchtein ID, Brouwer N, Sola PR, et al. Transcriptomic analysis of purified human cortical microglia reveals age-associated changes. Nat Neurosci. 2017;20:1162-71. https://doi.org/10.1038/nn.4597.

54. Gorina R, Font-Nieves M, Márquez-Kisinousky L, Santalucia T, Planas AM. Astrocyte TLR4 activation induces a proinflammatory environment through the interplay between MyD88-dependent NFkB signaling, MAPK, and Jak1/ Stat1 pathways. Glia. 2011;59:242-55. https://doi.org/10.1002/glia.21094.

55. Lehnardt S, Henneke P, Lien E, Kasper DL, Volpe JJ, Bechmann I, et al. A mechanism for neurodegeneration induced by group $B$ streptococci through activation of the TLR2/MyD88 pathway in microglia. J Immunol. 2006;177:583-92. https://doi.org/10.4049/jimmunol.177.1.583.

56. Okun E, Griffioen K, Barak B, Roberts NJ, Castro K, Pita MA, et al. Toll-like receptor 3 inhibits memory retention and constrains adult hippocampal neurogenesis. Proc Natl Acad Sci U S A. 2010;107:15625-30. https://doi.org/ 10.1073/pnas.1005807107.

57. Lathia JD, Okun E, Tang S-C, Griffioen K, Cheng A, Mughal MR, et al. Toll-like receptor 3 is a negative regulator of embryonic neural progenitor cell proliferation. J Neurosci. 2008;28:13978-84. https://doi.org/10.1523/ JNEUROSCI.2140-08.2008.

58. Tang S-C, Arumugam TV, Xu X, Cheng A, Mughal MR, Jo DG, et al. Pivotal role for neuronal Toll-like receptors in ischemic brain injury and functional deficits. Proc Natl Acad Sci U S A. 2007;104:13798-803. https://doi.org/10. 1073/pnas.0702553104.

59. Kawai T, Akira S. Toll-like receptors and their crosstalk with other innate receptors in infection and immunity. Immunity. 2011;34:637-50. https://doi. org/10.1016/j.jmmuni.2011.05.006

60. Alexopoulou L, Holt AC, Medzhitov R, Flavell RA. Recognition of doublestranded RNA and activation of NF-kappaB by Toll-like receptor 3. Nature. 2001;413:732-8. https://doi.org/10.1038/35099560.

61. Okun E, Griffioen KJ, Mattson MP. Toll-like receptor signaling in neural plasticity and disease. Trends Neurosci. 2011;34:269-81. https://doi.org/10. 1016/j.tins.2011.02.005.

62. Rolls A, Shechter R, London A, Ziv Y, Ronen A, Levy R, et al. Toll-like receptors modulate adult hippocampal neurogenesis. Nat Cell Biol. 2007;9: 1081-8. https://doi.org/10.1038/ncb1629.

63. Ma Y, Haynes RL, Sidman RL, Vartanian T. TLR8: an innate immune receptor in brain, neurons and axons. Cell Cycle. 2007;6:2859-68. https://doi.org/10. $4161 /$ cc.6.23.5018. 
64. Ma Y, Li J, Chiu I, Wang Y, Sloane JA, Lü J, et al. Toll-like receptor 8 functions as a negative regulator of neurite outgrowth and inducer of neuronal apoptosis. J Cell Biol. 2006;175:209-15. https://doi.org/10.1083/jcb. 200606016.

65. Cameron JS, Alexopoulou L, Sloane JA, DiBernardo AB, Ma Y, Kosaras B, et al. Toll-like receptor 3 is a potent negative regulator of axonal growth in mammals. J Neurosci. 2007;27:13033-41. https://doi.org/10.1523/JNEUROSCl. 4290-06.2007.

66. Crews FT, Qin L, Sheedy D, Vetreno RP, Zou J. High mobility group box 1/ Toll-like receptor danger signaling increases brain neuroimmune activation in alcohol dependence. Biol Psychiatry. 2013;73:602-12. https://doi.org/10. 1016/j.biopsych.2012.09.030.

67. Pandey GN, Rizavi HS, Ren X, Bhaumik R, Dwivedi Y. Toll-like receptors in the depressed and suicide brain. J Psychiatr Res. 2014;53:62-8. https://doi. org/10.1016/j.jpsychires.2014.01.021.

68. Pandey GN, Rizavi HS, Bhaumik R, Ren X. Innate immunity in the postmortem brain of depressed and suicide subjects: role of Toll-like receptors. Brain Behav Immun. 2019;75:101-11. https://doi.org/10.1016/j.bbi. 2018.09.024

69. García-Bueno B, Gassó P, MacDowell KS, Callado LF, Mas S, Bernardo M, et al. Evidence of activation of the Toll-like receptor-4 proinflammatory pathway in patients with schizophrenia. J Psychiatry Neurosci. 2016;41:E4655 http://www.ncbi.nlm.nih.gov/pubmed/27070349.

70. Pfefferbaum A, Rosenbloom M, Rohlfing T, Sullivan EV. Degradation of association and projection white matter systems in alcoholism detected with quantitative fiber tracking. Biol Psychiatry. 2009;65:680-90. https://doi. org/10.1016/j.biopsych.2008.10.039.

71. Najjar S, Pearlman DM. Neuroinflammation and white matter pathology in schizophrenia: systematic review. Schizophr Res. 2015;161:102-12. https:// doi.org/10.1016/j.schres.2014.04.041.

72. Shen X, Reus LM, Cox SR, Adams MJ, Liewald DC, Bastin ME, et al. Subcortical volume and white matter integrity abnormalities in major depressive disorder: findings from UK Biobank imaging data. Sci Rep. 2017;7: 5547. https://doi.org/10.1038/s41598-017-05507-6.

73. Song S-K, Sun S-W, Ju W-K, Lin S-J, Cross AH, Neufeld AH. Diffusion tensor imaging detects and differentiates axon and myelin degeneration in mouse optic nerve after retinal ischemia. Neuroimage. 2003;20:1714-22. https://doi. org/10.1016/j.neuroimage.2003.07.005.

74. Song S-K, Yoshino J, Le TQ, Lin S-J, Sun S-W, Cross AH, et al. Demyelination increases radial diffusivity in corpus callosum of mouse brain. Neuroimage. 2005;26:132-40. https://doi.org/10.1016/j.neuroimage.2005.01.028.

75. Sun S-W, Liang H-F, Trinkaus K, Cross AH, Armstrong RC, Song S-K Noninvasive detection of cuprizone induced axonal damage and demyelination in the mouse corpus callosum. Magn Reson Med. 2006;55: 302-8. https://doi.org/10.1002/mrm.20774

76. Nir TM, Jahanshad N, Villalon-Reina JE, Toga AW, Jack CR, Weiner MW, et al. Effectiveness of regional DTI measures in distinguishing Alzheimer's disease, MCl, and normal aging. Neurolmage Clin. 2013;3:180-95. https://doi.org/10. 1016/j.nicl.2013.07.006.

77. Lee SH, Coutu JP, Wilkens P, Yendiki A, Rosas HD, Salat DH. Tract-based analysis of white matter degeneration in Alzheimer's disease. Neuroscience. 2015;301:79-89. https://doi.org/10.1016/j.neuroscience.2015.05.049.

78. Liu Y, Spulber G, Lehtimäki KK, Könönen M, Hallikainen I, Gröhn H, et al. Diffusion tensor imaging and tract-based spatial statistics in Alzheimer's disease and mild cognitive impairment. Neurobiol Aging. 2011;32:1558-71. https://doi.org/10.1016/j.neurobiolaging.2009.10.006.

79. Hawrylycz M, Miller JA, Menon V, Feng D, Dolbeare T, Guillozet-Bongaarts $\mathrm{AL}$, et al. Canonical genetic signatures of the adult human brain. Nat Neurosci. 2015;18:1832-44. https://doi.org/10.1038/nn.4171.

\section{Publisher's Note}

Springer Nature remains neutral with regard to jurisdictional claims in published maps and institutional affiliations.

Ready to submit your research? Choose BMC and benefit from:

- fast, convenient online submission

- thorough peer review by experienced researchers in your field

- rapid publication on acceptance

- support for research data, including large and complex data types

- gold Open Access which fosters wider collaboration and increased citations

- maximum visibility for your research: over $100 \mathrm{M}$ website views per year

At $\mathrm{BMC}$, research is always in progress.

Learn more biomedcentral.com/submissions 